\title{
Dihydromyricetin Attenuates
} Dexamethasone-Induced Muscle Atrophy by Improving Mitochondrial Function via the PGC-1 $\alpha$ Pathway

\author{
Yujie Huang Ka Chen Qingbo Ren Long Yi Jundong Zhu Qianyong Zhang \\ Mantian Mi
}

Research Center for Nutrition and Food Safety, Chongqing Key Laboratory of Nutrition and Food Safety, Institute of Military Preventive Medicine, Third Military Medical University, Chongqing, China

\section{Key Words}

Dexamethasone $\cdot$ Muscle atrophy $\cdot$ Dihydromyricetin $\cdot$ Mitochondrial function $\cdot$ PGC-1 $\alpha$

\begin{abstract}
Background/Aims: Skeletal muscle atrophy is an important health issue and can impose tremendous economic burdens on healthcare systems. Glucocorticoids (GCs) are well-known factors that result in muscle atrophy observed in numerous pathological conditions. Therefore, the development of effective and safe therapeutic strategies for GC-induced muscle atrophy has significant clinical implications. Some natural compounds have been shown to effectively prevent muscle atrophy under several wasting conditions. Dihydromyricetin (DM), the most abundant flavonoid in Ampelopsis grossedentata, has a broad range of health benefits, but its effects on muscle atrophy are unclear. The purpose of this study was to evaluate the effects and underlying mechanisms of DM on muscle atrophy induced by the synthetic GC dexamethasone (Dex). Methods: The effects of DM on Dex-induced muscle atrophy were assessed in Sprague-Dawley rats and L6 myotubes. Muscle mass and myofiber cross-sectional areas were analyzed in gastrocnemius muscles. Muscle function was evaluated by a grip strength test. Myosin heavy chain (MHC) content and myotube diameter were measured in myotubes. Mitochondrial morphology was observed by transmission electron microscopy and confocal laser scanning microscopy. Mitochondrial DNA (mtDNA) was quantified by real-time PCR. Mitochondrial respiratory chain complex activities were examined using the MitoProfile Rapid Microplate Assay Kit, and mitochondrial membrane potential was assessed by JC-1 staining. Protein levels of mitochondrial biogenesis and dynamics markers were detected by western blotting. Myotubes were transfected with siRNAs targeting peroxisome proliferatoractivated receptor $\gamma$ coactivator $1 \alpha(\mathrm{PGC}-1 \alpha)$, mitochondrial transcription factor A (TFAM) and mitofusin-2 (mfn2) to determine the underlying mechanisms. Results: In vivo, DM preserved muscles from weight and average fiber cross-sectional area losses and improved grip strength. In vitro, DM prevented the decrease in MHC content and myotube diameter. Moreover, DM stimulated mitochondrial biogenesis and promoted mitochondrial fusion, rescued the
\end{abstract}


reduced mtDNA content, improved mitochondrial morphology, prevented the collapse in mitochondrial membrane potential and enhanced mitochondrial respiratory chain complex activities; these changes restored mitochondrial function and improved protein metabolism, contributing to the prevention of Dex-induced muscle atrophy. Furthermore, the protective effects of DM on mitochondrial function and muscle atrophy were alleviated by PGC-1 $\alpha$ siRNA, TFAM siRNA and mfn2 siRNA transfection in vitro. Conclusion: DM attenuated Dex-induced muscle atrophy by reversing mitochondrial dysfunction, which was partially mediated by the PGC-1 $\alpha /$ TFAM and PGC-1 $\alpha / \mathrm{mfn} 2$ signaling pathways. Our findings may open new avenues for identifying natural compounds that improve mitochondrial function as promising candidates for the management of muscle atrophy.

(C) 2018 The Author(s)

Published by S. Karger AG, Basel

\section{Introduction}

Skeletal muscle accounts for almost $40 \%$ of the total body mass, and the maintenance of healthy skeletal muscles is vital for providing energy for locomotion, preventing metabolic disorders and promoting healthy aging [1-3]. Many pathological conditions characterized by muscle atrophy, including cachexia, diabetes, sepsis, starvation, metabolic acidosis and chronic kidney disease, are associated with elevated circulating glucocorticoid (GC) levels [4]. Meanwhile, high doses or sustained administration of the exogenous synthetic GC dexamethasone (Dex) in clinical treatment causes muscle atrophy, which increases morbidity and mortality [5]. Thus, the identification of new targets and development of effective and safe therapeutic avenues are urgent medical needs to prevent GC-induced muscle atrophy.

The pivotal role of mitochondrial dysfunction in promoting muscle atrophy has been revealed in the past several years [6-11]. Recently, several studies demonstrated that mitochondrial dysfunction precedes and activates muscle atrophy signaling in Dex-induced muscle atrophy models [12] and some natural plant extracts, including hydroxytyrosol acetate, resveratrol and quercetin, can target the mitochondria to improve mitochondrial function and prevent muscle atrophy under several wasting conditions [12-14]. Thus, development of drugs or nutrients that improve mitochondrial function could be beneficial in preventing Dex-induced muscle atrophy. Ampelopsis grossedentata, a medicinal and edible plant growing in mountainous areas of southern China, has been consumed as a health tea and traditional medicine for hundreds of years [15]. Dihydromyricetin (DM) is the main flavonoid component of $A$. grossedentata, and the DM contents can be greater than $30 \%$ in vine tea made from its tender stems and leaves [16]. DM displays a broad range of biological and pharmacological activities such as antioxidant, anti-inflammatory, anti-tumor, neuroprotective and hepatoprotective effects [17-21]. Our previous studies demonstrated that DM could improve skeletal muscle insulin resistance and stimulate myokine secretion [22-24]. Importantly, we verified that DM could reverse mitochondrial dysfunction in skeletal muscle under acute hypoxic conditions, which contributes to improvements in physical performance [25]. However, no information is available regarding the effects of DM on skeletal muscle atrophy. The purpose of this study was to determine whether DM could improve mitochondrial function and attenuate Dex-induced muscle atrophy. The underlying mechanisms were also examined.

Mitochondrial biogenesis and dynamics are crucial for maintaining normal mitochondrial function [26]. Peroxisome proliferator-activated receptor $\gamma$ coactivator $1 \alpha$ (PGC-1 $\alpha$ ), a critical regulator of mitochondrial biogenesis, can control the expression of mitochondrial DNA (mtDNA)-encoded intramitochondrial proteins by regulating mitochondrial transcription factor A (TFAM) levels [27]. In addition, PGC-1 $\alpha$ has been shown to modulate mitochondrial dynamics by stimulating mitofusin ( $\mathrm{mfn} 1$ and $\mathrm{mfn} 2$ ) gene expression [28]. Importantly, the maintenance of high PGC-1 $\alpha$ levels in muscles preserves mitochondrial function and prevents muscle atrophy during disuse atrophy, sarcopenia, fasting and cachexia [29-32]. Previous studies have demonstrated that Dex suppressed PGC-1 $\alpha$ expression levels both in vivo and in vitro [33-34]; thus, treatments that preserve and increase PGC- $1 \alpha$ levels and signaling may be a plausible strategy to counteract Dex-induced muscle atrophy. We previously showed that DM treatment significantly increased the levels of PGC-1 $\alpha$ in skeletal muscle both in vivo and in vitro [24]. These findings led us to hypothesize that DM could attenuate Dex-induced muscle atrophy by reversing mitochondrial dysfunction through the PGC-1 $\alpha$ signaling pathway. As expected, our results confirmed, for the first time, that DM attenuated Dex-induced muscle atrophy by improving mitochondrial function, which was mediated in 


\section{Cellular Physiology Cell Physiol Biochem 2018:49:758-779

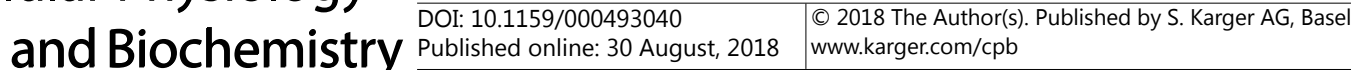

Huang et al.: Dihydromyricetin Improves Muscle Atrophy via PGC-1 $\alpha$

part by the PGC- $1 \alpha /$ TFAM and PGC- $1 \alpha / \mathrm{mfn} 2$ signaling pathways. These results indicated that DM treatment might be a promising approach for the prevention and treatment of Dexinduced muscle atrophy.

\section{Materials and Methods}

\section{Reagents and antibodies}

Cell Counting Kit-8 (CCk8; CK04) was purchased from Dojindo Laboratories (Kumamoto, Japan). Glass-bottom cell culture dishes (801001) were purchased from Nest Biotechnology (Wuxi, China). DM (mast-120131108, HPLC $\geq 98 \%$ ) was purchased from MUST Biotechnology Co., Ltd. (Chengdu, China). Dex (D4902), Dex (D1756), Akti (A6730), rapamycin (RAPA; R0395) and the Protein Carbonyl Content Assay Kit (MAK094) were purchased from Sigma-Aldrich (St. Louis, MO, USA). MitoTracker Deep Red (M22426) and MitoSOX Red (M36008) were purchased from Invitrogen (Carlsbad, CA, USA). Antibodies against myosin heavy chain (MHC; ab 24642), PGC-1 $\alpha$ (ab 106814), TFAM (ab 131607), OPA1 (ab 157457), Tom20 (ab 56783), mfn1 (ab 104585), mfn2 (ab 50838), Fox03a (ab 23683) and VDAC1 (ab 15895) were obtained from Abcam (Cambridge, UK). Antibodies against p-Akt (9271), Akt (9272), p-mTOR (2971), mTOR (2972), p-S6K (9205), S6K (9202) and GAPDH (5174) were obtained from Cell Signaling Technology (Beverly, MA). Antibodies against atrogin-1 (sc-33782) and MuRF1 (sc-27642) were obtained from Santa Cruz Biotechnology (Santa Cruz, CA, USA), whereas the antibody against drp1 (BD611113) was obtained from BD Biosciences (San Jose, CA).

\section{Animal studies}

Adult male Sprague Dawley (SD) rats $(n=50)$ with an average body weight of $200 \pm 24$ g were purchased from the Experimental Animal Center of the Third Military Medical University (Chongqing, China). Rats were housed in a specific pathogen free (SPF) grade room at a controlled temperature $\left(22 \pm 2{ }^{\circ} \mathrm{C}\right)$ with a 12 $\mathrm{h}$ light/dark cycle and had ad libitum access to food and water. All animal care and experimental procedures were conducted according to institutional guidelines with the approval of the Institutional Animal Care and Use Committee of the Third Military Medical University.

After acclimatization for 1 week, rats were randomized into five groups ( $\mathrm{n}=10$ per group): control group (Control); Dex-treated group (Dex); Dex plus $50 \mathrm{mg} / \mathrm{kg}$ body weight DM (DM50); Dex plus $100 \mathrm{mg} /$ kg body weight DM (DM100); Dex plus $200 \mathrm{mg} / \mathrm{kg}$ body weight DM (DM200). DM was dissolved in distilled water, and the Control group was given the same dose of distilled water as the placebo. DM was administered by gavage every day for 14 days prior to Dex treatment and maintained throughout the experimental period. Dex (500 $\mu \mathrm{g} / \mathrm{kg}$ body mass) was administered intraperitoneally once a day for 10 days. The Control group received an equal volume of normal saline. Body weight and food intake were assessed daily. Grip strength was measured with a grip strength meter (YuYan Instruments, Shanghai, China).

After the 24 day treatment, rats were sacrificed under anesthesia. Blood was collected and centrifuged at 3, $500 \mathrm{rpm}$ for $6 \mathrm{~min}$. The serum creatine kinase (CK) level was measured in an automatic analyzer (OLYMPUS AU5400, Japan). Gastrocnemius (GAS) and tibialis anterior (TA) muscles were quickly removed and collected for analyses.

\section{Measurement of cross-sectional area (CSA)}

Skeletal muscle tissues were dissected, fixed in fresh $4 \%$ paraformaldehyde solution and embedded in paraffin. For histological analysis, the paraffin sections were cut into slices $(5 \mu \mathrm{m})$ for hematoxylin and eosin (H\&E) staining. The images were captured with an Olympus VS120 microscope. The myofiber CSAs were determined for at least 500 fibers per GAS muscle, and the mean CSA was calculated. ImageJ software (NIH) was used for these analyses, which were conducted in a blinded fashion with the investigator being unaware of the group from which the images originated.

Observation of mitochondrial morphology using transmission electron microscopy (TEM)

Mitochondrial morphology in the GAS muscles was analyzed by TEM. GAS muscles from each group were trimmed into approximately $1.0 \mathrm{~mm}^{3}$ strips and fixed in $2.5 \%$ glutaraldehyde for $18-20 \mathrm{~h}$, followed by $1 \%$ osmium tetroxide for $1 \mathrm{~h}$. Then, the tissues were dehydrated, and embedded in epoxy. Ultra-thin sections (60 $\mathrm{nm}$ thick) were cut, and the sections were stained with $2 \%$ uranyl acetate and lead citrate and observed using a JEM-1400 microscope (JEOL, Japan). A minimum of 10 photomicrographs were taken randomly from each sample at 25, 000 $\times$ magnification. Three rats in each group and ten images per rat were used for the quantitative analysis of mitochondria. The number of mitochondria was counted on micrographs as previously described [35], and counting was performed in a blinded fashion. Meanwhile, we used the following criteria to classify mitochondria that were either normal or damaged. Mitochondria with 


\section{Cellular Physiology Cell Physiol Biochem 2018;49:758-779 and Biochemistry Published online: 30 August, 2018 \begin{tabular}{l|l} 
DOI: 10.1159/000493040 & $\begin{array}{l}\text { C } 2018 \text { The Author(s). Published by S. Karger AG, Basel } \\
\text { www.karger.com/cpb }\end{array}$
\end{tabular}}

Huang et al.: Dihydromyricetin Improves Muscle Atrophy via PGC-1 $\alpha$

any type of ultrastructural changes (broken outer and inner membranes, disrupted cristae, electron-dense mitochondria, intramitochondrial edema, and hypertrophic giant mitochondria) were considered damaged mitochondria. Then, the percentage of normal mitochondria was evaluated by the ratio between normal (healthy looking mitochondria) and all mitochondria.

\section{Cell culture and treatments}

Rat L6 myoblasts (obtained from ATCC-CRL-1458, Manassas, VA, USA) were cultured in Dulbecco's Modified Eagle's Medium (DMEM; Gibco-Invitrogen, Carlsbad, CA, USA) supplemented with 10\% fetal bovine serum (FBS; HyClone, USA) and 1\% penicillin-streptomycin in a $5 \% \mathrm{CO}_{2}$ humidified atmosphere at $37^{\circ} \mathrm{C}$. Then, the media were changed to DMEM containing $2 \%$ horse serum (Gibco, New Zealand) to induce myotube formation. When the cells differentiated to form myotubes, they were treated with Dex $(2 \mu \mathrm{M})$ in the presence or absence of DM $(10 \mu \mathrm{M})$ for $24 \mathrm{~h}$.

\section{Cell viability measurement}

Cell viability was measured by the CCK-8 detection kit as previously described [36]. Briefly, cells were cultivated in a 96-well microplate at a density of 8,000 cells/well and were differentiated to form myotubes. After exposure to the indicated treatments, twenty microliters of CCK-8 solution was added to each well, and the plate was incubated at $37^{\circ} \mathrm{C}$ for $2 \mathrm{~h}$. Cell viability was detected by absorbance measurements using an Infinite $^{T M}$ M200 Microplate Reader (Tecan, Mannedorf, Switzerland) at a wavelength of $450 \mathrm{~nm}$. The results are expressed as a percentage of the control.

\section{Cell transfection}

siRNAs for PGC-1 $\alpha$ (sc-72151), TFAM (sc-156067) and mfn2 (sc-156013) were purchased from Santa Cruz Biotechnology along with control siRNA (sc-44230) and siRNA Transfection Reagent (sc-29528). The siRNA for FoxO3a was synthesized by Shanghai GenePharma Co., Ltd. (Shanghai, China). L6 myotubes were transfected with $100 \mathrm{nM}$ siRNA for $6 \mathrm{~h}$ according to the manufacturer's protocol. Then, the myotubes were switched into fresh medium for an additional $24 \mathrm{~h}$. Where indicated, myotubes were treated with Dex and DM for $24 \mathrm{~h}$. Thereafter, myotubes were used for subsequent experiments.

\section{Measurement of myotube diameter}

For determination of myotube diameter, myotube cultures were photographed under a phase contrast microscope at $100 \times$ magnification. The myotube diameters were measured in a total of 60 myotubes from at least 10 random fields from each photograph using ImageJ software (NIH) as previously described [37]. The results were expressed as a percent of the control.

\section{Quantification of $m t D N A$}

mtDNA was extracted from GAS muscles and L6 myotubes using the Mito DNA Extraction Kit (Genmed Scientifics Inc., USA) according to the manufacturer's instructions. For the determination of the relative mtDNA copy number, the levels of mitochondrial ND1 (NADH-CoQ oxidoreductase 1) relative to the standard ACTB were evaluated by quantitative PCR using a qTower 2.2 real-time PCR system (Analytik Jena, German). The primer sequences are listed below: ND1 (forward: 5'-TTAATTGCCATGGCCTTCCTCACC-3'; reverse: 5'-TGGTTAGAGGGCGTATGGGTTCTT-3'); ACTB (forward: 5' - CCACCATGTACCCAGGCATT-3'; reverse: 5'-CGGACTCATCGTACTCCTGC-3'). Relative transcript abundance was calculated using the delta delta cycle threshold $(\Delta \Delta \mathrm{Ct})$ method.

\section{Measurement of oxidative phosphorylation complex activities and ATP levels}

Mitochondria were isolated from GAS muscles or myotubes using Mitochondria Isolation Kits (Beyotime, China) according to the manufacturer's protocol. The activities of complexes I, II, and IV and ATP synthase were assessed in mitochondrial homogenates of GAS muscles or myotubes using the MitoProfile Rapid Microplate Assay Kit (catalog nos. ab 109721, ab 109908, ab 109911, and ab 109714, respectively; Abcam, Cambridge, MA, USA) according to the manufacturer's instructions. ATP levels were measured in the whole muscle lysates using the ATP Assay Kit (Beyotime, China) following the manufacturer's instructions. The total and mitochondrial protein concentrations were determined using the BCA Protein Assay Kit (Beyotime, China).

\section{Measurement of MDA content and protein carbonyl levels}

MDA content was measured as an indicator of lipid oxidative damage in GAS muscles or myotubes using the MDA Detection Kit (Beyotime, China) according to the manufacturer's instructions. Protein carbonyl levels were determined as an indicator of protein oxidative damage in GAS muscles or myotubes 


\section{Cellular Physiology Cell Physiol Biochem 2018;49:758-779 \begin{tabular}{l|l|l} 
and Biochemistry & $\begin{array}{l}\text { DOI: 10.1159/000493040 } \\
\text { Published onlIne: 30 August, } 2018\end{array}$ & $\begin{array}{l}\text { C) 2018 The Author(s). Published by S. Karger AG, Basel } \\
\text { www.karger.com/cpb }\end{array}$ \\
\hline
\end{tabular}}

using the Protein Carbonyl Content Assay Kit following the manufacturer's protocol. The MDA content and protein carbonyl levels were expressed as nmol/mg protein.

\section{Measurement of mitochondrial oxygen consumption rate (OCR)}

L6 cells were seeded in XF Cell Culture Microplates. After differentiation and treatment, the cells were changed to unbuffered XF Base Medium supplemented with $1 \mathrm{mM}$ pyruvate, $2 \mathrm{mM}$ glutamine and $10 \mathrm{mM}$ glucose and incubated at $37^{\circ} \mathrm{C}$ in a non-CO2 incubator for $1 \mathrm{~h}$. Then, the OCR was measured by the Extracellular Flux Analyzer (Seahorse Bioscience, Massachusetts, USA) as previously described [38]. The data were analyzed using Wave and Report Generator software.

\section{Measurement of mitochondrial membrane potential}

Mitochondrial membrane potential $(\Delta \psi \mathrm{m})$ was assessed in GAS muscles or myotubes using the JC-1 probe (Beyotime, China). Red fluorescence represents JC-1 J-aggregates, whereas green fluorescence represents the monomeric form of JC-1. For muscle tissues, isolated mitochondria were immediately suspended in a JC-1 staining solution and analyzed by an Infinite ${ }^{T M}$ M200 Microplate Reader following the manufacturer's instructions. The $\Delta \psi \mathrm{m}$ in each group was calculated as the ratio of red/green fluorescence.

For myotubes, cells were cultured in a cell culture dish. After the indicated treatment, cells were incubated with a JC-1 staining solution for $20 \mathrm{~min}$ at $37^{\circ} \mathrm{C}$. Images were obtained using a Zeiss confocal laser scanning microscopy (Carl Zeiss, LSM 800). The red and green fluorescence intensities were quantified using the Zeiss LSM Image Examiner software. The $\Delta \psi \mathrm{m}$ was reflected by the ratio of red to green fluorescence intensity. The results were obtained from at least 30 cells in each of three experiments per group.

\section{Visualization of mitochondrial network in L6 myotubes}

Cells were grown on coverslips inside a cell culture dish. After treatment, the culture medium in the dish was replaced with prewarmed staining solution containing $250 \mathrm{nM}$ MitoTracker Deep Red probe. Cells were incubated with the staining solution for $30 \mathrm{~min}$ at $37^{\circ} \mathrm{C}$. After staining was complete, the cells were washed two times with prewarmed PBS and incubated with fresh prewarmed media. Then, the cells were visualized using a Zeiss confocal laser scanning microscope. Mitochondrial mass was analyzed by quantifying the mitochondrial fluorescence intensity using Zeiss LSM Image Examiner software. The results were obtained from at least 30 cells in each of three experiments per group.

Measurement of reactive oxygen species (ROS) levels and mitochondrial-derived $\mathrm{O}_{2}^{-{ }^{-}}$levels in L6 myotubes

Total levels of cellular ROS in L6 myotubes were determined using the ROS Assay Kit (Beyotime, China) according to the manufacturer's instructions. For the analysis of mitochondrial $\mathrm{O}_{2}{ }^{--}$, L6 myotubes were labeled with MitoSOX Red, a highly selective fluorescent probe, for the detection of $\mathrm{O}_{2}{ }^{\cdot-}$ generated within mitochondria. Briefly, cells were cultured in a cell culture dish and were treated as indicated. MitoSOX Red reagent stock solution was diluted in $\mathrm{HBSS} / \mathrm{Ca} / \mathrm{Mg}$ buffer to generate a $5 \mu \mathrm{M}$ reagent working solution. Cells were then incubated with $5 \mu \mathrm{M}$ MitoSOX Red reagent working solution in the dark at $37{ }^{\circ} \mathrm{C}$ for $10 \mathrm{~min}$. After three washes with warm buffer, live imaging was performed using a Zeiss confocal laser scanning microscope. The fluorescence intensity was quantified using the Zeiss LSM Image Examiner software. The results were obtained from at least 30 cells in each of three experiments per group.

Western blot analysis

GAS muscle tissues were homogenized on ice in a Tissue Protein Extraction Reagent supplemented with a protease inhibitor (Roche Applied Science, Germany). Myotubes were homogenized on ice in RIPA lysis buffer (Beyotime, China) supplemented with PMSF. For the analysis of mitochondrial proteins, the isolated mitochondria from GAS muscles or myotubes were homogenized in the mitochondrial lysis buffer supplemented with PMSF (Beyotime, China). The lysates were centrifuged at 12, $000 \mathrm{~g}$ for $20 \mathrm{~min}$ at 4 ${ }^{\circ} \mathrm{C}$ and the supernatants were collected. Then, the protein samples were subjected to $10-15 \%$ SDS-PAGE, transferred to PVDF membranes (Bio-Rad), blocked with $5 \%$ skim milk for $2 \mathrm{~h}$, incubated overnight at $4{ }^{\circ} \mathrm{C}$ with primary antibodies and then incubated with horseradish peroxidase-conjugated secondary antibodies for $1 \mathrm{~h}$. Protein bands were visualized using an enhanced chemiluminescence system (Millipore, Billerica, MA, USA) and densitometric analysis was performed using Quantity One software (Bio-Rad Lab., Hercules, CA, USA).

\section{Statistical analysis}

SPSS 13.0 statistical software (SPSS Inc., Chicago, IL) was used for statistical analyses. Quantitative data are expressed as the mean \pm standard deviations (SD). Statistical comparisons were conducted using one-way analysis of variance (ANOVA) followed by Tukey's post hoc test for multiple comparisons. $P<0.05$ was considered statistically significant. 


\section{Cellular Physiology Cell Physiol Biochem 2018:49:758-779

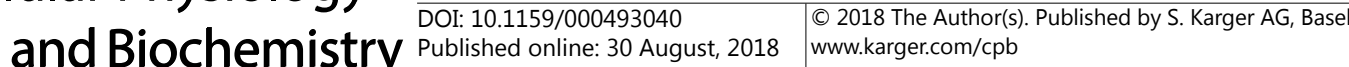

Huang et al.: Dihydromyricetin Improves Muscle Atrophy via PGC-1 $\alpha$

\section{Results}

DM attenuated Dex-induced muscle atrophy and dysfunction in SD rats

The baseline body weight was similar in each group. After treatment, Dex caused a significant decrease in the body weight compared to the control group, while the DM-200 group had a higher final body weight than the Dex-group (Fig. 1a). In addition, there was no significant difference in the average food intake between the Dex-group and DM-treated groups. Consistent with the decrease in body weight, Dex caused a marked decline in the weights of both the GAS and TA muscles as well as the muscle/body weight ratio compared to the control group (Fig. 1b and 1c). The DM-100 and DM-200 groups had higher GAS muscle weights and GAS muscle/body weight ratios than the Dex-group, and the DM-200 group had higher TA muscle weights and TA muscle/body weight ratio than the Dex-group (Fig. $1 \mathrm{~b}$ and $1 \mathrm{c}$ ). Histologically, Dex induced a significant decrease in the average myofiber CSA in GAS muscles, while the DM-100 and DM-200 groups exhibited an increase in the average fiber CSA of the GAS muscles compared to the Dex-group (Fig. 1d and 1e). These results suggested that DM treatment preserved GAS muscles from weight and average fiber CSA loss. Paralleling the changes in muscle mass, muscle function, assessed by grip strength, was improved in the DM-200 group compared to the Dex-group (Fig. 1f). Additionally, the serum levels of CK, a well-known biomarker of muscle damage, were increased by Dex but were reduced in the DM-100 and DM-200 groups compared to the Dex-group (Fig. 1g). Taken together, these results suggested that DM treatment attenuated Dex-induced muscle atrophy and dysfunction in SD rats.

\section{DM attenuated Dex-induced mitochondrial injuries and modulated related protein} expression

The mitochondrial morphology changes in the intermyofibrillar mitochondria (IFM) were observed by TEM. The Dex-group showed swollen and atypical mitochondrial with irregular and fragmented cristae, while DM treatment efficiently counteracted the mitochondrial morphological abnormalities, with less swollen mitochondrial and more intact cristae compared to the Dex-group (Fig. 2a). The quantitative analysis of mitochondria revealed that the number of IFM in the Dex-group decreased compared to the control group, while the number of IFM in the DM-100 and DM-200 groups increased compared to the Dexgroup (Fig. 2b). Similar results were observed for the percentage of normal mitochondria (Fig. 2c).

To further elucidate the alterations in mitochondrial morphology and number, we evaluated mitochondria-related protein expression. Levels of PGC-1 $\alpha$ and TFAM, key molecules related to mitochondrial biogenesis, were decreased by Dex but were increased in the DM-200 group compared to the Dex-group (Fig. $2 \mathrm{~d}$ and 2e). Similar results were observed for $\mathrm{mfn} 2$ expression (Fig. $2 \mathrm{~d}$ and $2 \mathrm{e}$ ). However, $\mathrm{mfn} 1$ and the mitochondrial fission protein drp1 were not affected by Dex or DM (Fig. 2d and 2e). Additionally, OPA1 expression was downregulated in the Dex-group compared to that in the control group, while no obvious changes in the levels of the OPA1 protein were observed after DM treatment (Fig. $2 \mathrm{~d}$ and $2 \mathrm{e}$ ). We also quantified the mitochondria-related protein levels in the mitochondrial subfractions. Consistent with the changes in TFAM, OPA1, $\mathrm{mfn} 1$ and $\mathrm{mfn} 2$ protein contents in the whole muscle lysates, the levels of TFAM and $\mathrm{mfn} 2$ in the mitochondrial subfractions were increased in the DM-200 group compared to the Dex-group, and the OPA1 and mfn1 levels remained unchanged upon treatment with DM (Fig. $2 \mathrm{f}$ and $2 \mathrm{~g}$ ). Inconsistent with the changes in drp1 levels in the whole muscle lysates, the levels of drp1 in the mitochondrial subfractions were increased by Dex but were reduced in the DM-100 and DM-200 groups compared to the Dex-group (Fig. 2f and 2g). Moreover, the expression of Tom20, an accepted mitochondrial marker protein, was decreased upon Dex treatment but was increased in the DM-200 group compared to the Dex-group (Fig. $2 \mathrm{~h}$ and 2i). Furthermore, treatment with $\mathrm{DM}$ at 100 and $200 \mathrm{mg} / \mathrm{kg}$ body weight significantly inhibited Dex-induced loss of mtDNA content as evidenced by the restoration of the mtDNA/nDNA ratio (Fig. 2j).

DM suppressed the collapse of mitochondrial membrane potential, protected against reduced activities of mitochondrial respiratory chain complexes, and alleviated oxidative stress in skeletal muscle tissues

We then investigated the effects of DM on mitochondrial function in GAS muscles. The results showed that Dex induced the loss of mitochondrial membrane potential $(\Delta \psi \mathrm{m})$ 


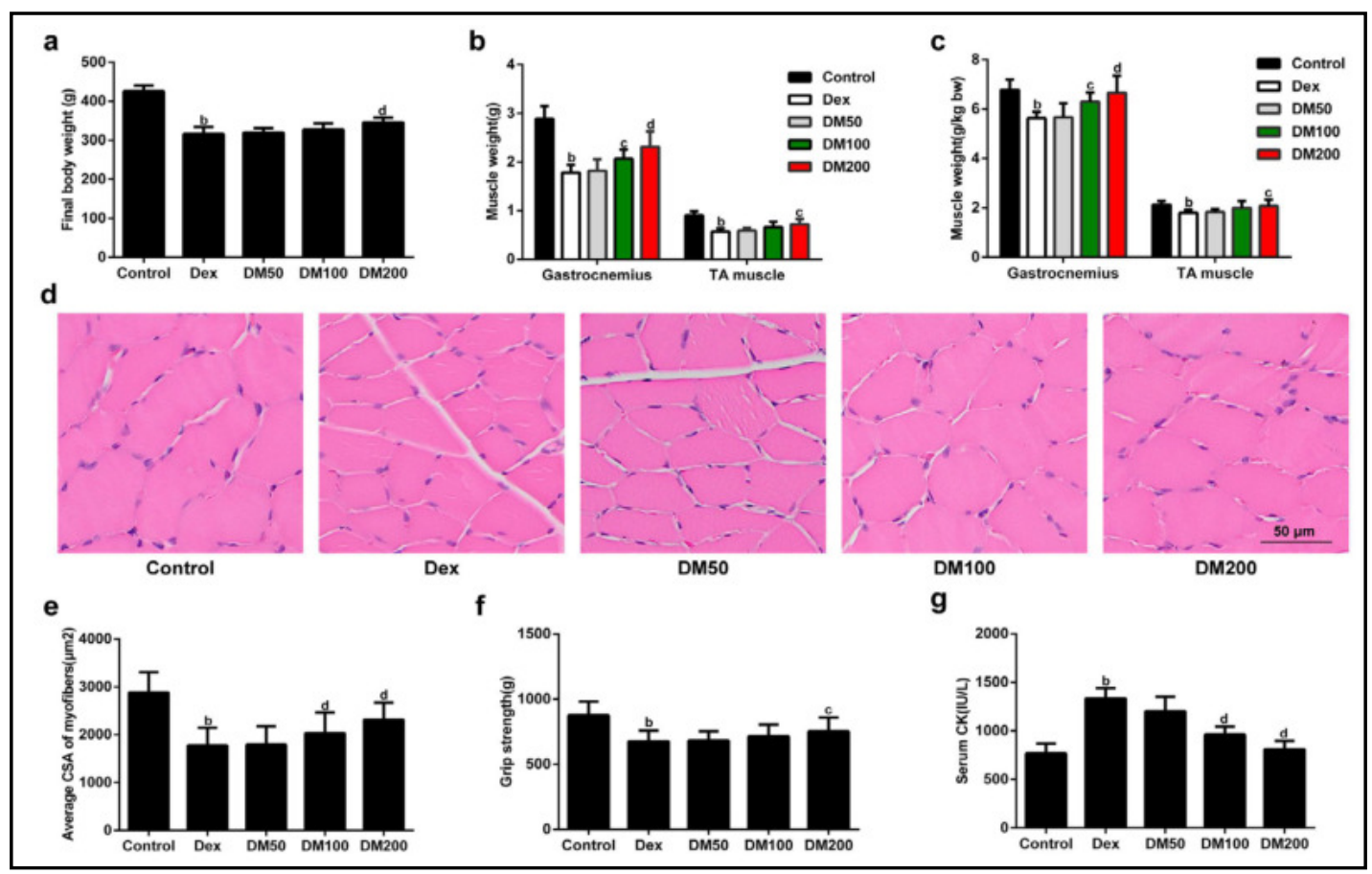

Fig. 1. DM prevents Dex-induced muscle atrophy in SD rats. (a) Final body weight in each group. (b) Weights of GAS and TA muscles in each group. (c) The ratio of muscle weight to body weight. (d) Representative microscopy images of H\&E staining of GAS muscle sections. Scale bar, $50 \mu \mathrm{m}$. (e) Quantitation of average muscle CSA of GAS in each group. (f) Grip strength of the rats during treatment in each group. (g) Serum CK levels were measured after sacrifice. Data are expressed as the mean $\pm \mathrm{SD}(\mathrm{n}=10) .{ }^{\mathrm{b}} \mathrm{P}<0.01$ vs. control group; ${ }^{c} \mathrm{P}<0.05$, ${ }^{\mathrm{d}} \mathrm{P}<0.01$ vs. Dex-treated group.

indicated by a decrease in the red/green fluorescent ratio, while DM treatment at $200 \mathrm{mg} /$ $\mathrm{kg}$ body weight repressed the changes of $\Delta \psi \mathrm{m}$ induced by Dex (Fig. 3a). Moreover, the Dexgroup had lower complex I, II, and IV and ATP synthase activities than the control group, while the DM-200 group had higher activities of all the above complexes (Fig. 3b-e). Similar results were observed for ATP levels (Fig. 3f).

Mounting evidence suggests that mitochondria are the primary source of ROS[39], and reduced activities of the mitochondrial respiratory chain complexes can result in the overproduction of mitochondrial ROS (mtROS) at the expense of ATP production [40]. Moreover, excessive mtROS production can promote oxidative stress and cause oxidative damage in tissues [41]. To explore the effects of Dex and DM on oxidative stress in GAS muscles, we examined the protein carbonyl levels as markers for protein oxidative damage and MDA content for lipid oxidative damage. The data showed that DM treatment at 100 and $200 \mathrm{mg} / \mathrm{kg}$ body weight markedly prevented Dex-induced elevation of protein carbonyl levels and MDA content (Fig. $3 \mathrm{~g}$ and $3 \mathrm{~h}$ ). Together, these results suggested that DM treatment efficiently attenuated Dex-induced muscle mitochondrial dysfunction in vivo.

\section{DM attenuated Dex-induced muscle atrophy in vitro}

To confirm the findings obtained in SD rats, we further examined the effects of DM on Dexinduced muscle atrophy in L6 myotubes. As shown in Fig. 4a, at a Dex concentration of $2 \mu \mathrm{M}$, the inhibition of cell survival was approximately $19 \%$. Additionally, Dex-mediated inhibition of cell survival was significantly alleviated by DM in a dose-dependent manner. Moreover, Dex induced a substantial decrease in the myofibrillar protein MHC expression, which was dose-dependently prevented by DM (Fig. 4b and 4c). The greatest protective effect of DM was observed at the $10 \mu \mathrm{M}$ dose; thus, we used this dose in subsequent experiments. Furthermore, Dex reduced the myotube diameter by approximately $26 \%$, which was also prevented by DM (Fig. $4 \mathrm{~d}$ ). Together, these results suggested that DM efficiently protected against Dex-induced cell death and muscle atrophy in vitro. 
Fig. 2. Effect of DM on mitochondrial structure and related protein expression in the GAS muscles of rats. (a) Representative TEM images of the IFM at an original magnification of 25,000×. Scale bar, $1 \mu \mathrm{m}$. The presence of mitochondria with degraded or swollen cristae (black arrows) is shown in micrographs. (b) Quantification of the number of mitochondria per section. (c) Quantified results of the percentage of normal mitochondria. (d) Representative images of the western blotting results for PGC- $1 \alpha$, TFAM, OPA1, mfn1, mfn2 and drp1 in the whole muscle lysates; GAPDH was used as a loading control. (e) The bar charts show quantification of the indicated proteins. (f)

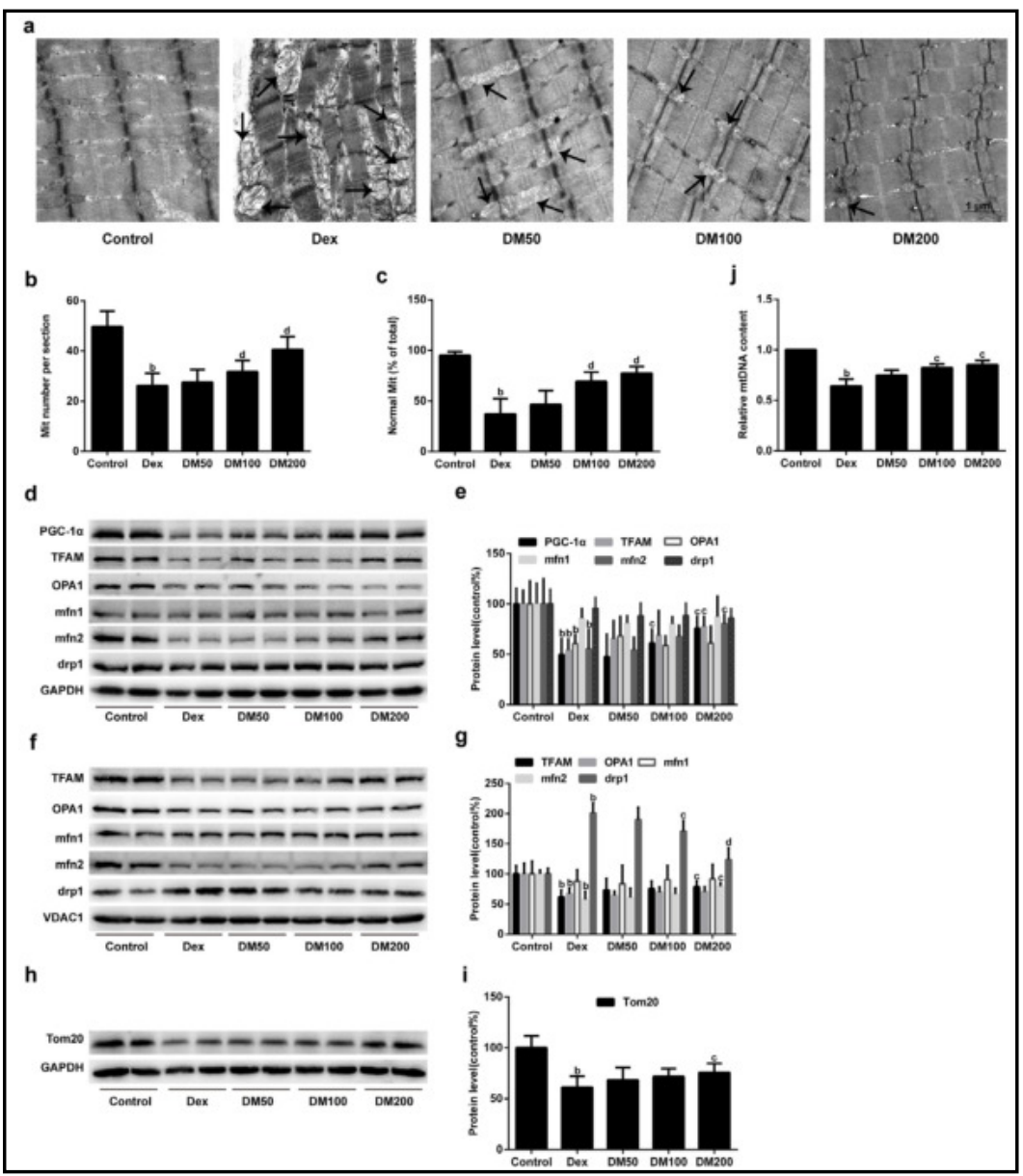
Representative images of the western blotting results for TFAM, OPA1, $\mathrm{mfn} 1, \mathrm{mfn} 2$ and drp1 in the mitochondrial subfractions; VDAC1 was used as a loading control. (g) The bar charts show quantification of the indicated proteins. (h) Representative image of the western blotting results for Tom20 in the GAS muscles; GAPDH was used as a loading control. (i) The bar graph shows quantification of Tom20. (j) Relative mtDNA copy number in the GAS muscles. Data are expressed as the mean $\pm \mathrm{SD}(\mathrm{n}=3) .{ }^{\mathrm{b}} \mathrm{P}<0.01$ vs. control group; ${ }^{\mathrm{c}} \mathrm{P}<0.05,{ }^{\mathrm{d}} \mathrm{P}<0.01$ vs. Dex-treated group.

DM improved mitochondrial morphology, rescued the reduced mtDNA content and repressed Dex-induced collapse of mitochondrial membrane potential $(\Delta \psi m)$ in vitro

To assess the effects and underlying mechanisms of DM on mitochondrial function during muscle atrophy in vitro, we first evaluated the related protein expression. As shown in Fig. 5a-b and 5e-f, DM dose-dependently restored the Dex-mediated reductions in PGC-1 $\alpha$, TFAM, mfn2 and Tom 20 protein levels and inhibited Dex-mediated increased expression of drp1, while it had no significant effect on mfn 1 and OPA1 expression. Moreover, PGC- $1 \alpha$ siRNA transfection markedly inhibited DM-induced PGC- $1 \alpha$ activation accompanied by decreased expression of TFAM and mfn2, while the expression of drp1 was not affected (Fig. $5 \mathrm{c}$ and 5d). Similar results were observed for TFAM, mfn2 and drp1 levels in the mitochondrial subfractions (Fig. 5g and $5 \mathrm{~h}$ ). These results suggested that DM activated the PGC-1 $\alpha /$ TFAM and PGC- $1 \alpha / \mathrm{mfn} 2$ signaling pathways. To determine whether the PGC- $1 \alpha /$ TFAM and PGC$1 \alpha / \mathrm{mfn} 2$ signaling pathways mediated the effects of DM on the mitochondria, we applied PGC-1 $\alpha$ siRNA, TFAM siRNA and mfn2 siRNA on L6 myotubes. The effects of downregulation of TFAM and mfn 2 by specific siRNAs were evaluated in the mitochondrial subfractions. As shown in Fig. 5i-l, the TFAM and mfn2 levels after the siRNA treatments were significantly lower compared to those of the control siRNA-treated group, suggesting that the siRNAs successfully inhibited TFAM and $\mathrm{mfn} 2$ expression. 


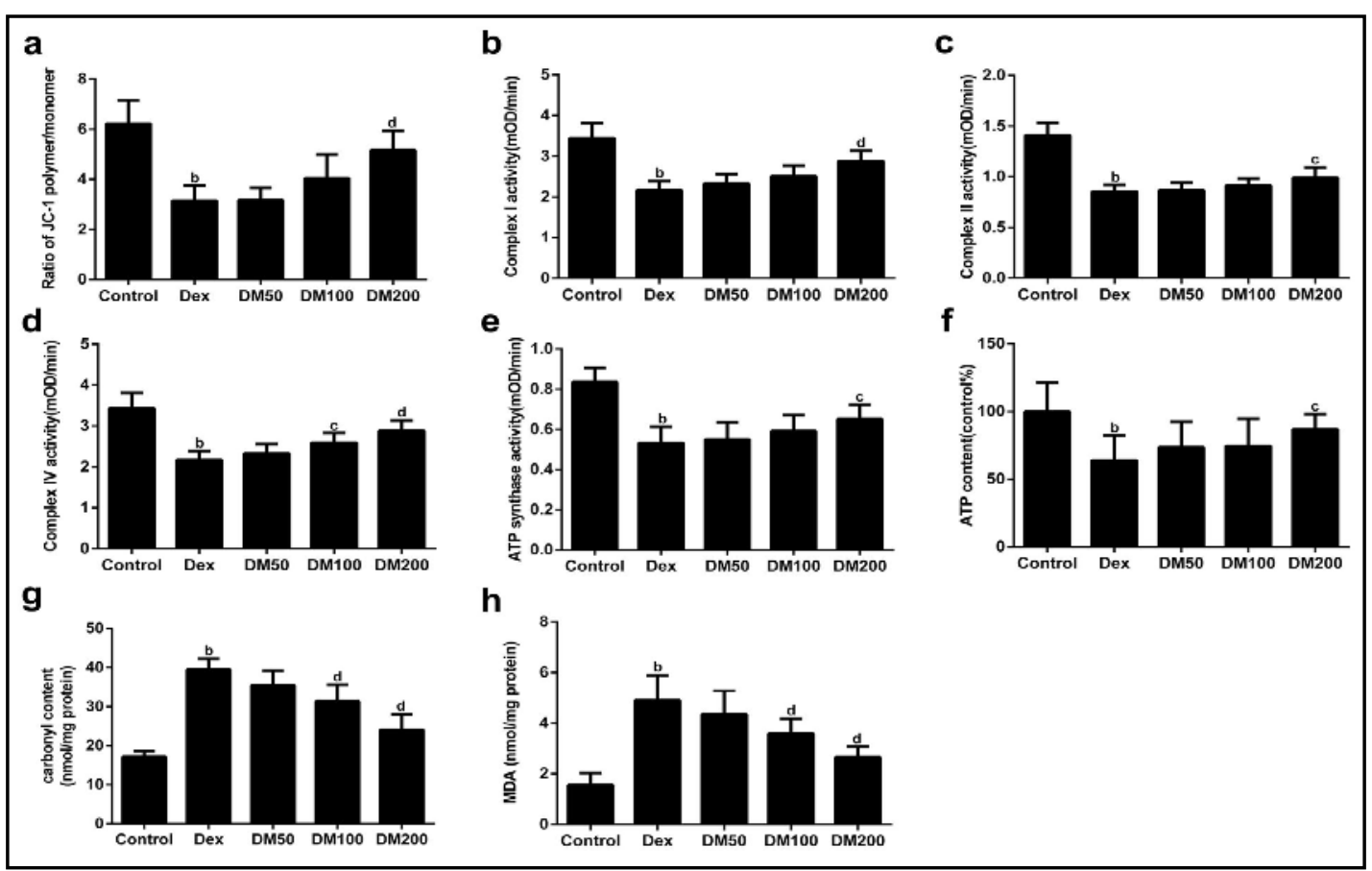

Fig. 3. Effect of DM on mitochondrial membrane potential $(\Delta \psi \mathrm{m})$, mitochondrial respiratory chain activity, ATP content, protein carbonylation level and MDA content in the GAS muscles. (a) $\Delta \psi \mathrm{m}$ in each group was calculated as the ratio of red/green fluorescence. (b, c, d, e) Activities of complexes I, II, and IV and ATP synthase. (f) ATP content. (g) Muscle protein carbonyl levels. (h) Muscle MDA content. Data are expressed as the mean $\pm \mathrm{SD}(\mathrm{n}=10)$. ${ }^{\mathrm{b}} \mathrm{P}<0.01$ vs. control group; ${ }^{\mathrm{C}} \mathrm{P}<0.05,{ }^{\mathrm{d}} \mathrm{P}<0.01$ vs. Dex-treated group.

To gain insight into the mitochondrial morphology, we incubated L6 myotubes with MitoTracker probes. As shown in Fig. 6a, the mitochondria displayed elongated and filamentous morphology in the control group. Following Dex treatment, the mitochondrial architecture was dramatically altered from that of the control group as demonstrated by punctate and highly fragmented mitochondria with a discontinuous network. DM treatment blocked Dex-induced excessive mitochondrial fragmentation and increased the filamentous mitochondria. In a further analysis, we found that Dex reduced mitochondrial mass, which was prevented by DM treatment (Fig. 6b). However, PGC- $1 \alpha$ siRNA, TFAM siRNA and mfn2 siRNA transfection abolished these effects of DM (Fig. 6a and 6b). Similar results were observed for the mtDNA content (Fig. 6c).

Next, we measured the effects of DM on $\Delta \psi \mathrm{m}$ in L6 myotubes using the JC-1 probe. As shown in Fig. 6d and 6e, Dex decreased the ratio of red to green fluorescence, indicating the depolarization of $\Delta \psi \mathrm{m}$, while DM treatment significantly repressed Dex-induced dissipation of $\Delta \psi \mathrm{m}$. However, PGC- $1 \alpha$ siRNA, TFAM siRNA and $\mathrm{mfn} 2$ siRNA transfection abolished the effect of DM on Dex-induced changes of $\Delta \psi \mathrm{m}$. Together, these results suggested that DM improved mitochondrial morphology, rescued the reduced mtDNA content and repressed the collapse of $\Delta \psi \mathrm{m}$ through the PGC- $1 \alpha / \mathrm{TFAM}$ and PGC- $1 \alpha / \mathrm{mfn} 2$ signaling pathways in vitro.

DM ameliorated mitochondrial dysfunction and suppressed oxidative stress in vitro

We measured oxygen consumption rates (OCR) to confirm the hypothesis that mitochondrial function was increased by DM treatment in Dex-treated myotubes in vitro. The results showed that Dex reduced the mitochondrial respiration capacity, including basal respiration, ATP potential and maximal respiration, all of which were improved by DM treatment (Fig. 7a and 7b). However, PGC-1 $\alpha$ siRNA, TFAM siRNA and mfn2 siRNA transfection impaired DM-induced mitochondrial respiratory recovery (Fig. 7a and 7b). Then, the activities of mitochondrial respiratory chain complexes in L6 myotubes were measured. As shown in Fig. 7c-f, Dex significantly decreased the activities of complexes I, II, and IV and ATP synthase, while DM treatment restored the activities of complexes I and IV 
Fig. 4. DM attenuates Dex-induced muscle atrophy in L6 myotubes. (a) Myotubes were treated with 2 $\mu \mathrm{M}$ Dex in the presence or absence of different concentrations $(0.1,1$, and $10 \mu \mathrm{M}$ ) of DM for $24 \mathrm{~h}$. Cell viability was measured using a CCK-8 assay, as described in the Materials and Methods section. (b) Representative image of the western blotting results for MHC; GAPDH was used as a loading control. (c) The bar graph shows quantification of MHC. (d) Quantification of myotube diameter, as described in the Materials and Methods section. Data are expressed as the mean

\section{a}

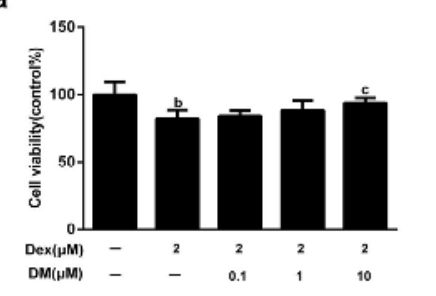

C

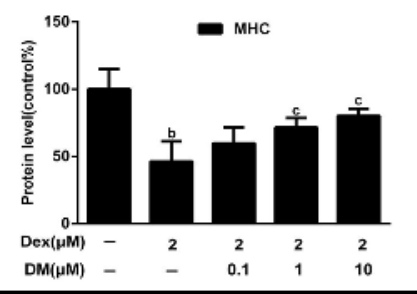

b

d

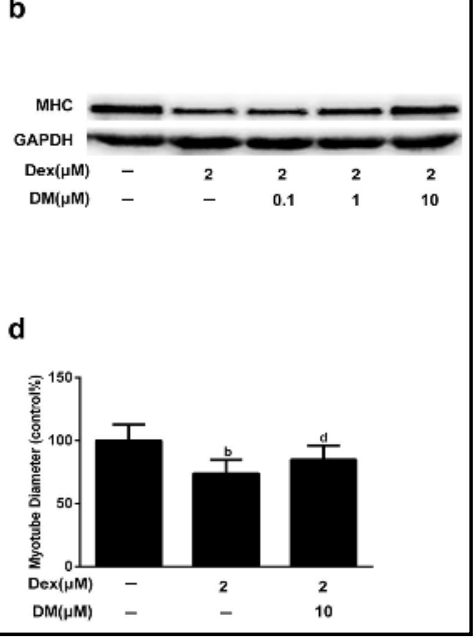
$\pm \mathrm{SD}(\mathrm{n}=3) .{ }^{\mathrm{b}} \mathrm{P}<0.01$ vs. control group; ${ }^{\mathrm{C}}<0.05$, ${ }^{\mathrm{d}} \mathrm{P}<0.01$ vs. Dex-treated group.

and ATP synthase. DM treatment also tended to restore the activity of complex II, although there was no significant difference between any DM-group and Dex-group. However, PGC-1 $\alpha$ siRNA, TFAM siRNA and mfn2 siRNA transfection abolished the stimulatory effects of DM on the activities of complexes I and IV and ATP synthase. Similar alterations were observed in ATP levels using the corresponding assay kits (Fig. 7g).

Next, we measured intracellular ROS levels using the fluorescent probe DCFH-DA. As shown in Fig. 7h, Dex markedly increased cellular ROS levels, which was alleviated by DM treatment. However, PGC- $1 \alpha$ siRNA, TFAM siRNA and mfn 2 siRNA transfection abolished the effect of DM on ROS levels. The superoxide anion $\left(\mathrm{O}_{2}{ }^{-}\right)$generated in mitochondria is the primary ROS; thus, we evaluated the mitochondrial-derived $\mathrm{O}_{2}{ }^{-{ }^{-}}$levels in myotubes labeled with MitoSOX Red to investigate the effect of DM on mtROS production. As shown in Fig. $7 \mathrm{i}$ and $7 \mathrm{j}$, DM treatment notably blocked Dex-induced mitochondrial-derived $\mathrm{O}_{2}{ }^{--}$elevation in myotubes. However, this effect of DM was sharply abolished by PGC- $1 \alpha$ siRNA, TFAM siRNA and mfn2 siRNA transfection. Similar results were observed for the protein carbonyl levels and MDA content (Fig. 7k and 7l). Together, these results suggested that DM reversed mitochondrial dysfunction through PGC- $1 \alpha /$ TFAM and PGC- $1 \alpha / \mathrm{mfn} 2$ signaling pathways in vitro.

DM inhibited FoxO3a-mediated protein degradation and increased Akt/mTOR pathway

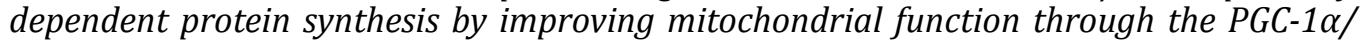
TFAM and PGC-1 $\alpha / m f n 2$ signaling pathways

Previous studies have revealed that FoxO3a plays a pivotal role in the skeletal muscle atrophy program, as it modulates the expression of several factors in the ubiquitin-proteasome proteolytic pathway [42]. To determine whether DM regulates these critical components associated with muscle atrophy, we examined the protein levels of FoxO3a and the specific ubiquitin ligases, atrogin-1 and MuRF1. Treatment with DM at 100 and $200 \mathrm{mg} / \mathrm{kg}$ body weight markedly inhibited the Dex-mediated increased expression of Fox03a, atrogin- 1 and MuRF1 in vivo (Fig. 8a and 8b). Similar results were observed in L6 myotubes (Fig. 8c and 8d). Moreover, the decrease in MHC content, as well as the increase in atrogin-1 and MuRF1 expression upon Dex treatment, were all normalized by FoxO3a siRNA transfection (Fig. 8e and 8f). To further characterize the mechanisms mediating the effects of DM on Dex-induced muscle atrophy models, we measured the Akt/mTOR signaling pathway, a crucial pathway regulating protein synthesis and muscle hypertrophy [43]. Both in vivo and in vitro results demonstrated that treatment with DM restored the Dex-mediated reductions in p-Akt and p-mTOR protein levels (Fig. 9a-d). Moreover, DM also reactivated S6K (Fig. 9a-d), an effector in protein synthesis and a downstream signaling molecule in the Akt/mTOR pathway [44]. Furthermore, pretreatment with Akti (Akt inhibitor) and rapamycin (mTOR inhibitor) markedly blocked the DM-induced increased expression of MHC and p-S6K (Fig. 9e and 9f). These data indicated that DM inhibited protein degradation by inactivating FoxO3a and 

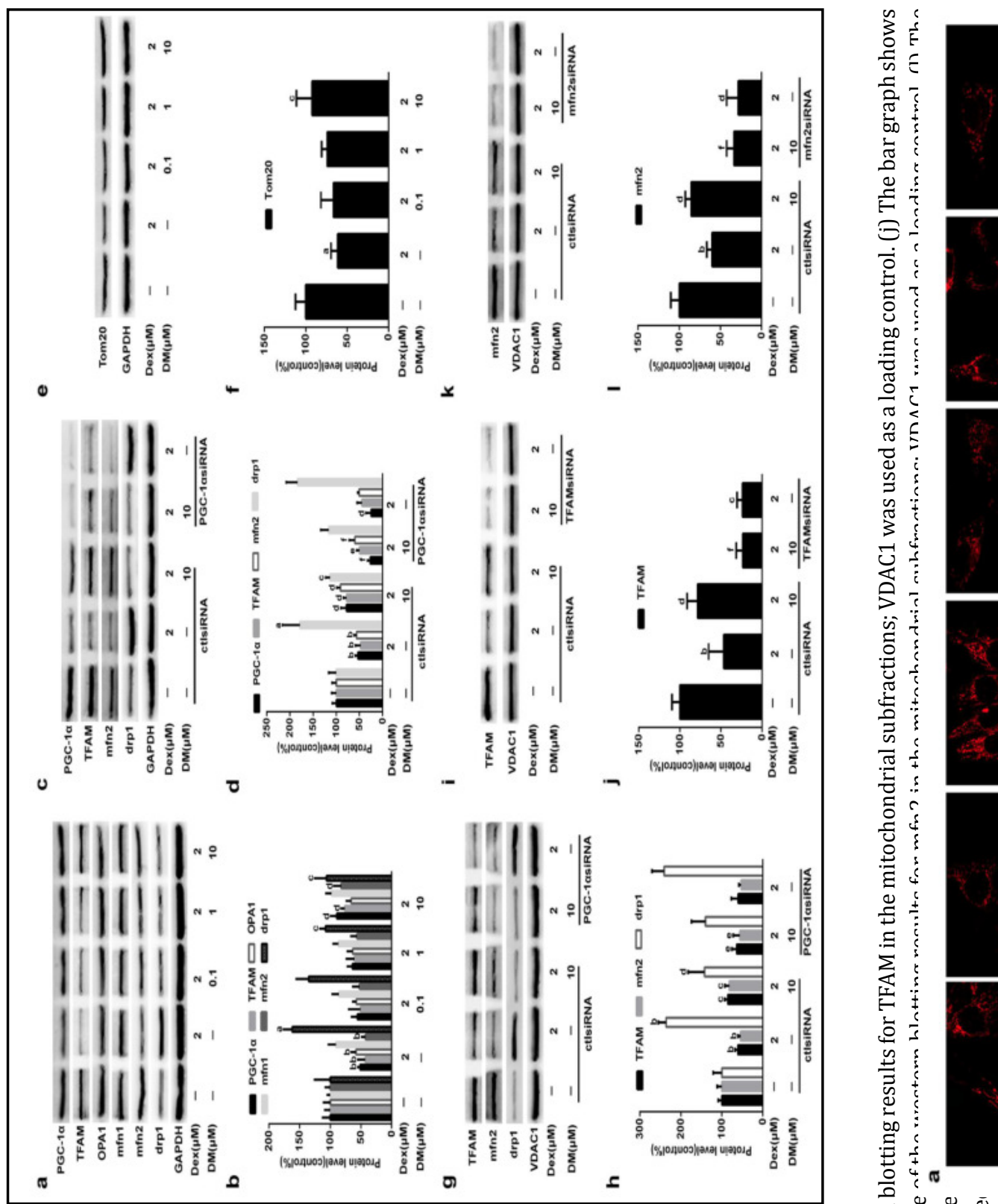

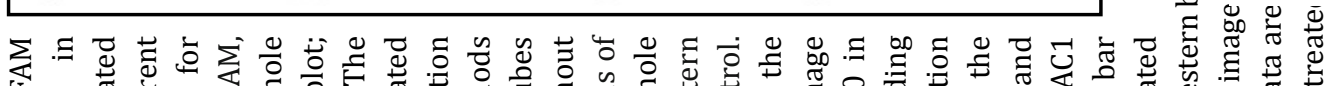

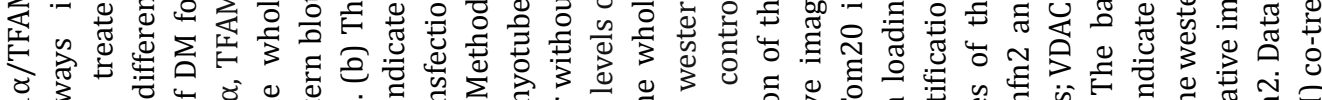

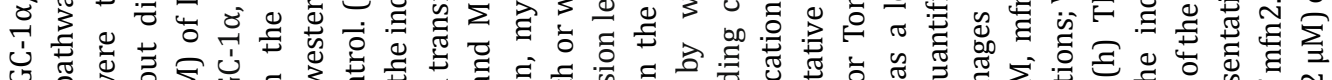

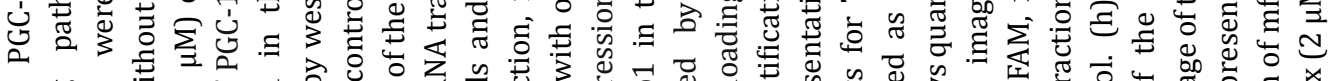

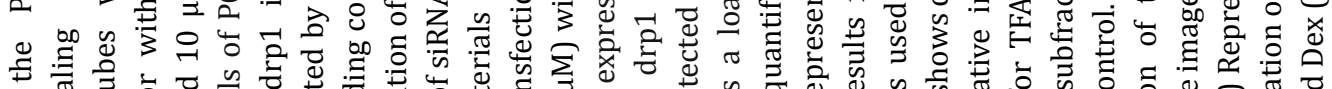
元

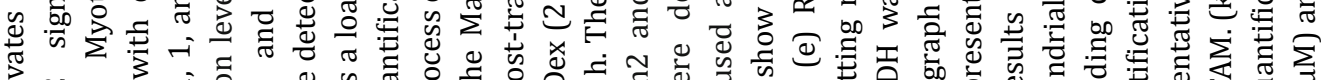

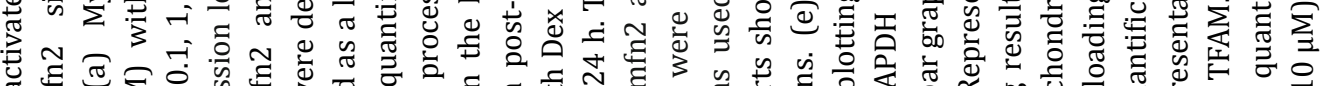

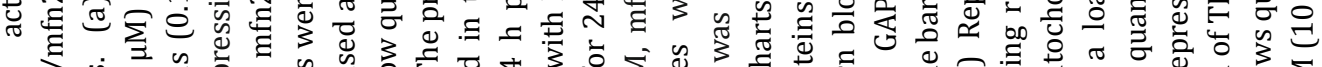

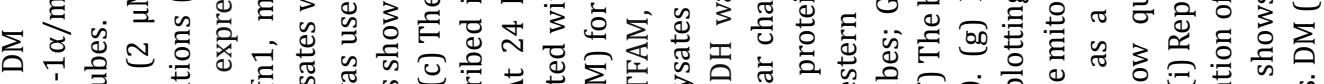

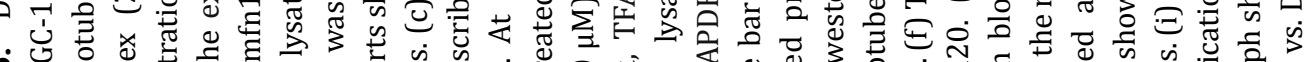

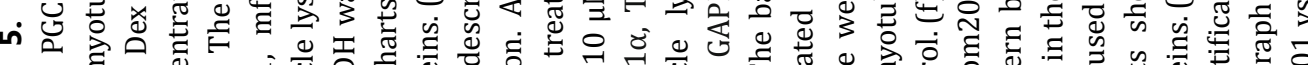

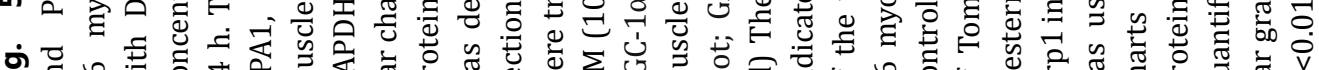

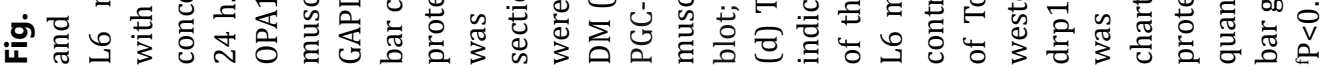
KARGER 


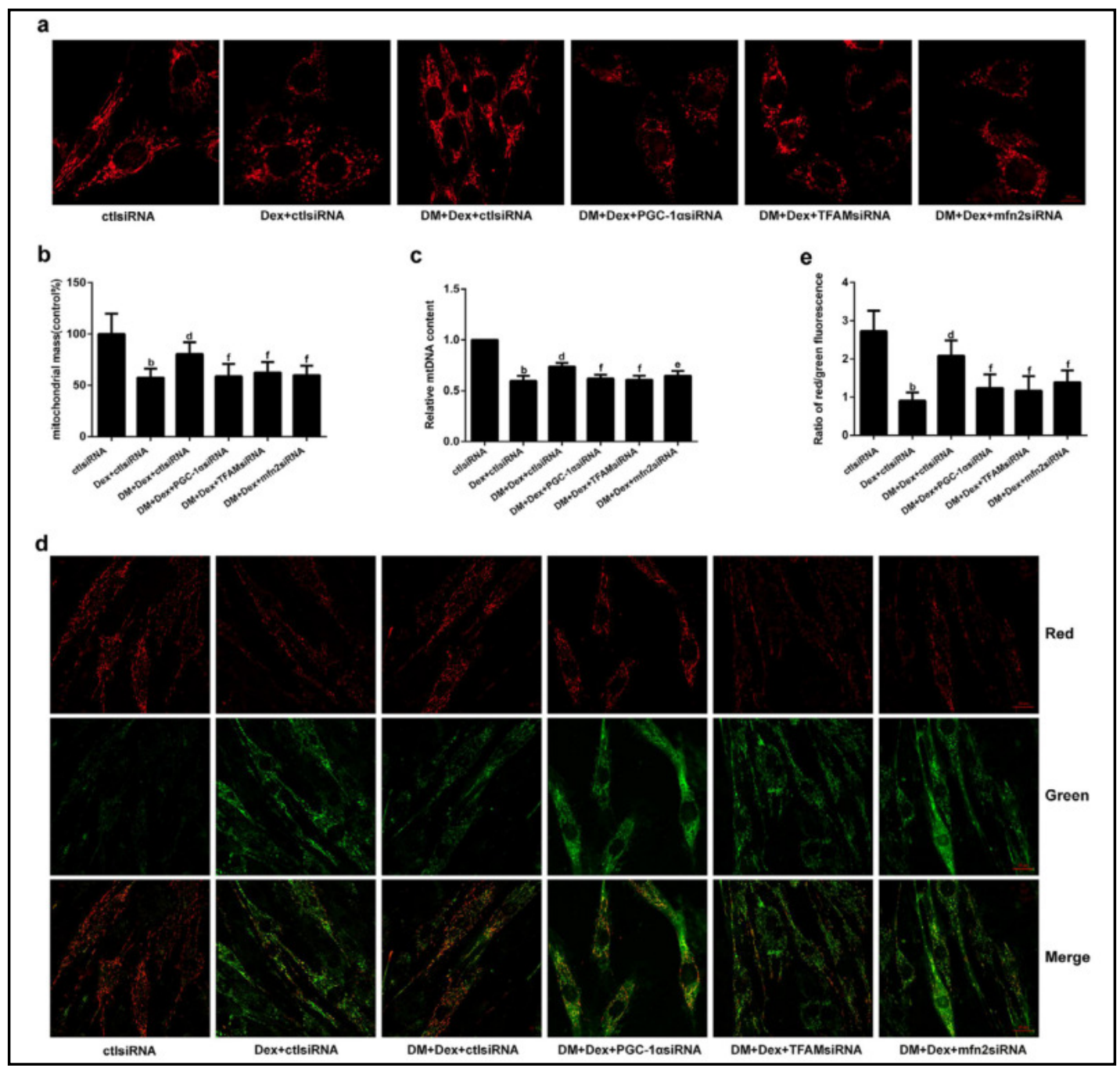

Fig. 6. Effect of DM on mitochondrial morphology, mtDNA content, and mitochondrial membrane potential $(\Delta \psi \mathrm{m})$ in L6 myotubes. (a) Mitochondrial morphology was visualized by MitoTracker probes. Scale bar, $10 \mu \mathrm{m}$. (b) Mitochondrial mass was analyzed by calculating the fluorescent intensity of MitoTracker Red. (c) Relative mtDNA copy number in L6 myotubes. (d) Determination of $\Delta \psi \mathrm{m}$ in L6 myotubes using the JC-1 probe. Red fluorescence represents the mitochondrial aggregate form of JC-1, indicating healthy mitochondria with intact mitochondrial membrane. Green fluorescence represents the monomeric form of JC-1, indicating mitochondrial membrane depolarization. The merged images indicate co-localization of JC-1 aggregates and monomers. Scale bar, $20 \mu \mathrm{m}$. (e) The ratio of red fluorescence to green fluorescence. Data are expressed as the mean $\pm \mathrm{SD}(\mathrm{n}=3)$. ${ }^{\mathrm{b}} \mathrm{P}<0.01$ vs. control group; ${ }^{\mathrm{P}}<0.01$ vs. Dex-treated group; ${ }^{\mathrm{e}} \mathrm{P}<0.05$, ${ }^{\mathrm{f}} \mathrm{P}<0.01$ vs. DM $(10 \mu \mathrm{M})$ and Dex $(2 \mu \mathrm{M})$ co-treated group with control siRNA transfection.

increased protein synthesis through the activation of AKT/mTOR pathway.

Growing evidence suggests that mitochondrial dysfunction plays an important role in protein metabolism [6]. Therefore, we hypothesized that the protective effects of DM on muscle protein metabolism were associated with its role in improving mitochondrial function through the PGC- $1 \alpha /$ TFAM and PGC- $1 \alpha / \mathrm{mfn} 2$ signaling pathways. As expected, PGC- $1 \alpha$ siRNA, TFAM siRNA and mfn2 siRNA transfection abrogated the effects of DM on Fox03a, atrogin-1, MuRF1, p-Akt, p-mTOR and p-S6K levels in myotubes (Fig. 8c and 8d). Moreover, the protective effects of DM on the Dex-induced MHC decline were abolished by PGC-1 $\alpha$ siRNA, TFAM siRNA and mfn2 siRNA transfection (Fig. 8c and 8d).

Overall, we concluded that mitochondrial dysfunction upon Dex treatment led to reduced protein synthesis and enhanced ubiquitin-proteasome-mediated protein catabolism and that DM improved protein metabolism and attenuated Dex-induced muscle atrophy by 


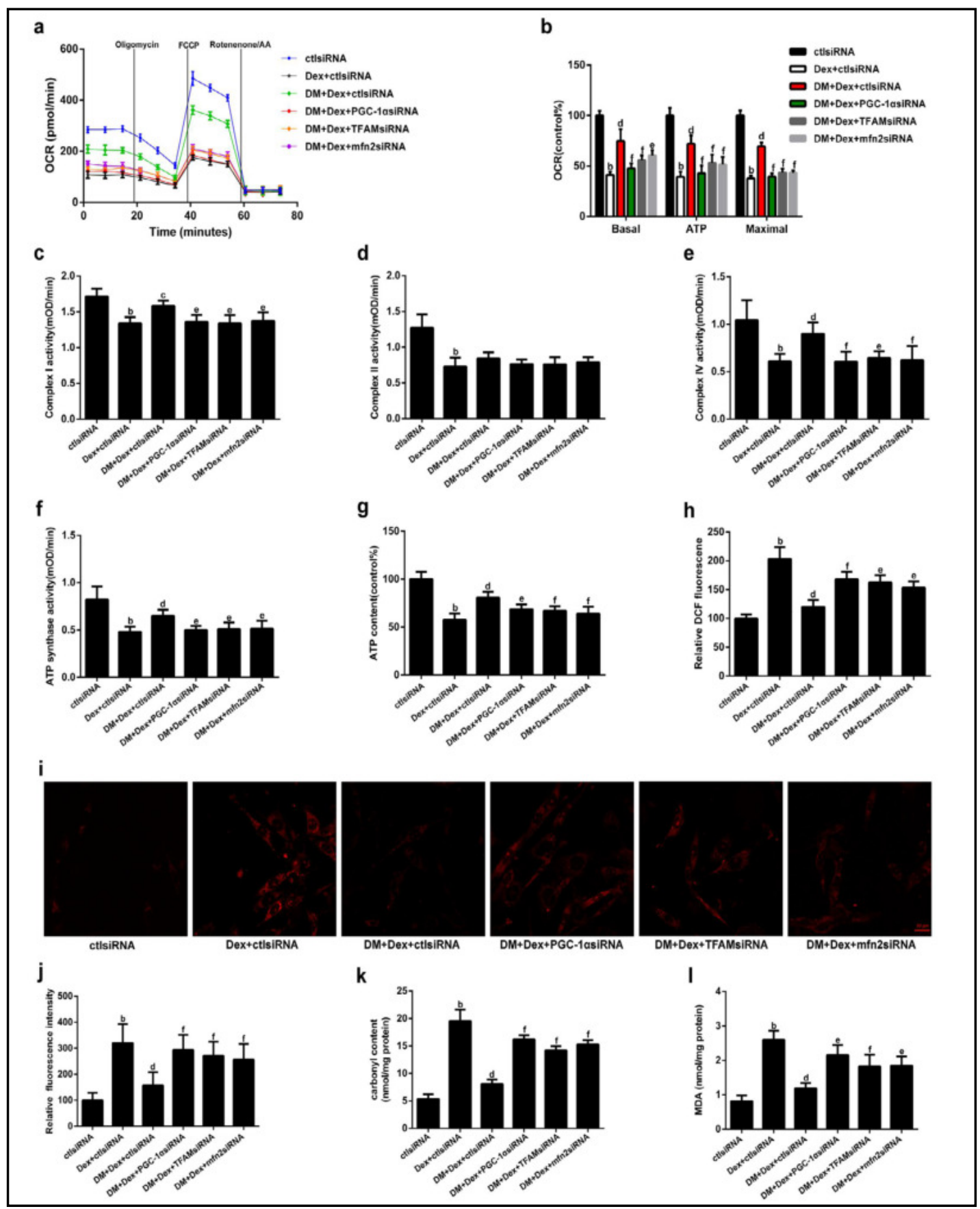

Fig. 7. Effect of DM on OCR, mitochondrial respiratory chain activity, ATP content and oxidative stress in L6 myotubes. (a) The respiration curve in each group. (b) The bar charts show quantification of basal respiration, ATP potential and maximal respiration. (c, d, e, f) Activities of complexes I, II, and IV and ATP synthase. (g) ATP content. (h) Intracellular ROS levels were detected using the fluorescent probe DCFH-DA. (i) The mitochondrial $\mathrm{O}_{2}^{--}$levels were detected in myotubes labeled with MitoSOX Red by confocal laser scanning microscopy. Scale bar, $20 \mu \mathrm{m}$. (j) The bar charts show quantification of fluorescence intensity. (k) Protein carbonyl levels in L6 myotubes. (l) MDA content in L6 myotubes. Data are expressed as the mean \pm $\mathrm{SD}(\mathrm{n}=3)$. ${ }^{\mathrm{b}} \mathrm{P}<0.01$ vs. control group; ${ }^{\mathrm{C}} \mathrm{P}<0.05$, ${ }^{\mathrm{d}} \mathrm{P}<0.01$ vs. Dex-treated group; ${ }^{\mathrm{e}} \mathrm{P}<0.05$, $\mathrm{P}<0.01$ vs. $\mathrm{DM}(10 \mu \mathrm{M})$ and Dex $(2 \mu \mathrm{M})$ co-treated group with control siRNA transfection.

reversing mitochondrial dysfunction through the PGC- $1 \alpha /$ TFAM and PGC- $1 \alpha / \mathrm{mfn} 2$ signaling pathways. 
Fig. 8. $\mathrm{DM}$ inhibited Fox 03 a-dependent atrophy programme by improving mitochondrial function through the PGC$1 \alpha /$ TFAM and PGC- $1 \alpha /$ $\mathrm{mfn} 2$ signaling pathways. (a) Representative images of the western blotting results for Fox03a, atrogin-1 and MuRF1 in the GAS muscles; GAPDH was used as a loading control. (b) The bar charts show quantification of the indicated proteins. (c) Representative images of the western blotting results for FoxO3a, atrogin-1, MuRF1 and MHC in L6 myotubes; GAPDH was used as a loading control. (d) The bar charts show quantification of the indicated proteins. (e) Representative images of the western blotting results for FoxO3a,

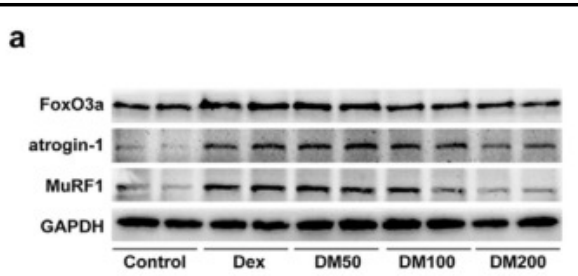

b

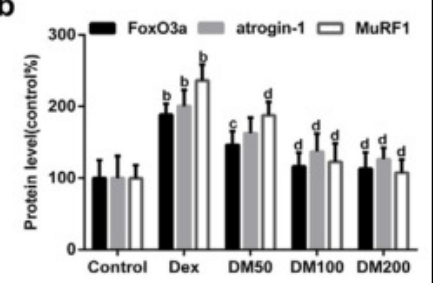

c

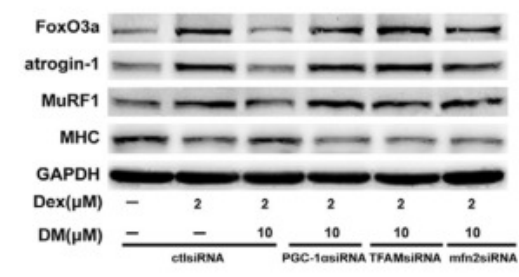

d

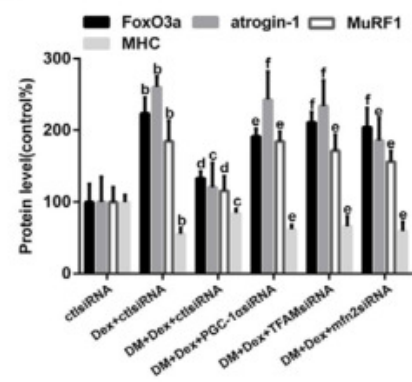

f

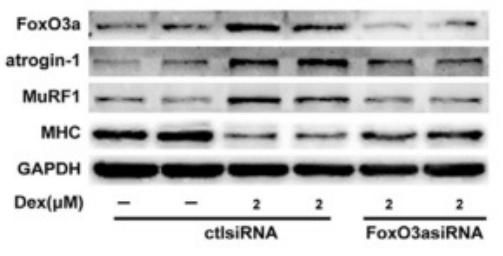
atrogin-1, MuRF1 and

MHC in L6 myotubes; GAPDH was used as a loading control. (f) The bar charts show quantification of the indicated proteins. Data are expressed as the mean $\pm \mathrm{SD}(\mathrm{n}=3) .{ }^{\mathrm{b}} \mathrm{P}<0.01$ vs. control group; ${ }^{\mathrm{C}} \mathrm{P}<0.05,{ }^{\mathrm{d}} \mathrm{P}<0.01$ vs. Dex-treated group; ${ }^{\mathrm{e}} \mathrm{P}<0.05$, ${ }^{\mathrm{f}} \mathrm{P}<0.01$ vs. $\mathrm{DM}(10 \mu \mathrm{M})$ and $\mathrm{Dex}(2 \mu \mathrm{M})$ co-treated group with control siRNA transfection.

\section{Discussion}

Skeletal muscle plays a crucial role in human locomotion, energy metabolism, and the release of multiple types of myokines [45]. Excessive loss of muscle mass can compromise physical function, impair the efficacy of many different therapeutic treatments, and increase morbidity and mortality [3]. Thus, the development of countermeasures to block or attenuate muscle atrophy will improve quality of life and survival. Although several potentially interesting therapeutic targets for treating muscle atrophy have already been identified, an effective and safe drug that can counteract muscle atrophy is not yet clinically available [3]. Drugs such as ghrelin, clenbuterol and testosterone have been shown to prevent muscle atrophy under several wasting conditions, but their clinical applications are limited because of concerns regarding potential side effects, such as an increased risk of developing prostate hypertrophy, insulin resistance, and cardiac arrhythmias [46]. Therefore, further research is needed to develop effective and safe therapeutic avenues for muscle atrophy.

Natural products, mostly from plant sources, contain a range of bioactive components and have a long history in the traditional treatment of many diseases. Considering that many medicinal plants and natural products are considered by the public as a safe and cost-effective alternative to synthetic drugs [47], natural product libraries may be a valuable source of preventive and therapeutic agents for muscle atrophy. Flavonoids, which exist ubiquitously in vegetables, fruits, teas, and medicinal plants, possess a wide range of health benefits [48]. In recent years, the effects of flavonoids on muscle atrophy under various conditions have received increased attention. Quercetin has been shown to prevent obesity and disuse- 
Fig. 9. DM increased Akt/ mTOR pathway dependent protein synthesis by improving mitochondrial function through the PGC$1 \alpha /$ TFAM and PGC- $1 \alpha /$ mfn2 signaling pathways. (a) Representative images of the western blotting results for p-Akt, Akt, p-mTOR, mTOR, p-S6K and S6K in the GAS muscles; GAPDH was used as a loading control. (b) The bar charts show the relative protein levels. (c) Representative images of the western blotting results for p-Akt, Akt, p-mTOR, mTOR, p-S6K and S6K in L6 myotubes; GAPDH was used as a loading control. (d) The bar charts show the relative protein levels. (e) Myotubes were preincubated with Akti $(10 \mu \mathrm{M})$ or rapamycin $(0.1 \mu \mathrm{M})$ for $1 \mathrm{~h}$ followed by the addition of Dex and DM for $24 \mathrm{~h}$. The expression of p-Akt, Akt, p-mTOR, mTOR, p-S6K, S6K and MHC was a

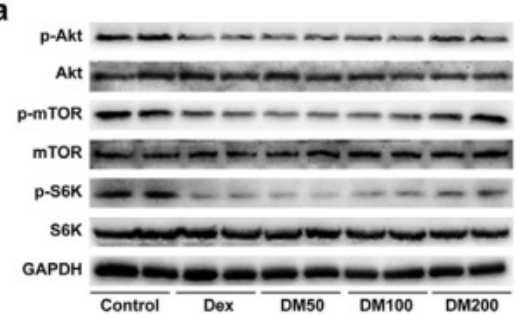

C

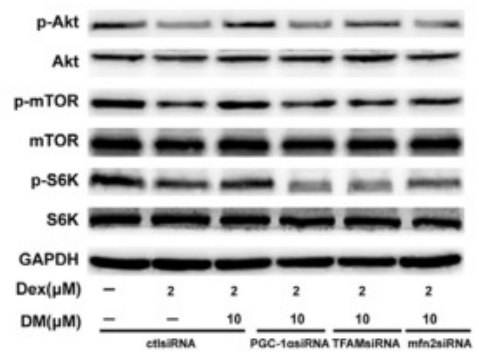

e

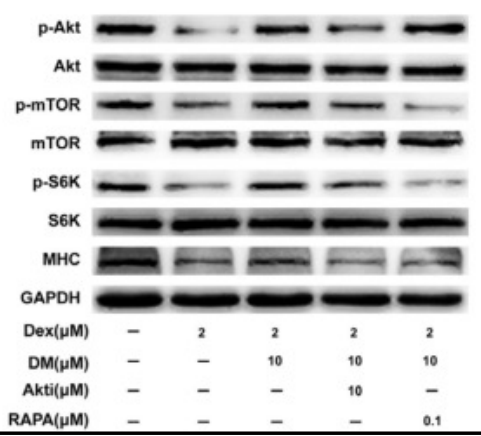

b

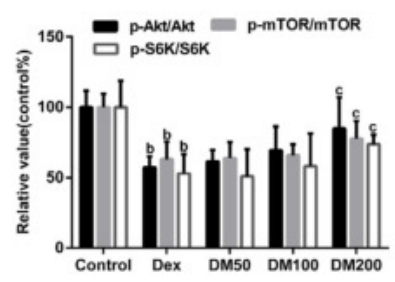

d

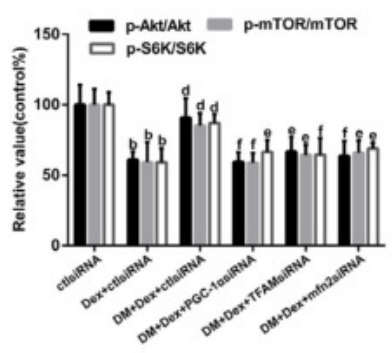

f

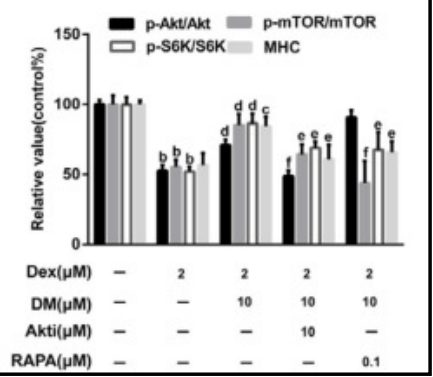

measured by western blot; GAPDH was used as a loading control. (f) The bar charts show the relative protein levels. Data are expressed as the mean $\pm \mathrm{SD}(\mathrm{n}=3)$. ${ }^{\mathrm{b}} \mathrm{P}<0.01 \mathrm{vs}$. control group; ${ }^{\mathrm{c}} \mathrm{P}<0.05,{ }^{\mathrm{d}} \mathrm{P}<0.01$ vs. Dex-treated group; ${ }^{\mathrm{e}} \mathrm{P}<0.05$, $\mathrm{f}<0.01$ vs. $\mathrm{DM}(10 \mu \mathrm{M})$ and $\mathrm{Dex}(2 \mu \mathrm{M})$ co-treated group.

induced muscle atrophy [49-50]. Baicalin and epigallocatechin-3-gallate were reported to attenuate skeletal muscle atrophy caused by cancer cachexia [51-53]. A recent study suggested that delphinidin could suppress Dex-induced MuRF1 expression in myotubes [54]. In the present study, we focused on DM, a promising natural compound for food use, due to its abundance, broad range of health benefits and long history of safe consumption [17]. Our previous studies showed that supplementation of DM for 2 weeks had no significant effect on body weight and muscle weight in normal SD rats. To investigate the effects of DM on Dex-induced muscle atrophy, DM was administered for 2 weeks prior to Dex treatment as a preventive approach. The results suggested that DM treatment partially prevented muscle loss and myofiber CSA decrease in Dex-treated rats, which contributed to the restoration of muscle strength by DM. Moreover, the decreases in MHC content and myotube diameter, as well as the increase in ubiquitin E3 ligases (atrogin-1/MuRF1) expression in Dextreated myotubes, were all sufficiently normalized by DM treatment. These data identified new properties of DM, namely, the ability of DM to attenuate Dex-induced muscle atrophy. Furthermore, our study provided new insight into the potential mechanisms underlying the protective effects of DM on Dex-induced muscle atrophy, in which mitochondrial function regulation may play an important role.

GCs play a crucial role in muscle atrophy observed in various pathological conditions [55]. Importantly, a recent study indicated that mitochondrial dysfunction has a central role in Dex-induced muscle atrophy models and that resveratrol can reverse muscle atrophy by improving mitochondrial function [12]. Mitochondrial function is affected by changes in morphology and abundance [7]. In this study, ultrastructural observation by TEM indicated 
that DM treatment attenuated Dex-induced mitochondrial swell and cristae disarray in GAS muscles, and the mitochondrial network visualized by MitoTracker probes showed that DM treatment conferred resistance to mitochondrial fragmentation in myotubes. Moreover, both in vivo and in vitro results demonstrated that DM treatment prevented the decrease in the mtDNA content. Concomitant with these changes, mitochondrial function, reflected by mitochondrial membrane potential, mitochondrial respiratory chain complex activities and ATP levels, were all improved by DM in Dex-induced muscle atrophy models. Our results demonstrated clear evidence that DM reversed Dex-induced mitochondrial dysfunction, which was crucial for preventing Dex-induced muscle atrophy. We further examined the mechanisms by which DM exerted its action on mitochondrial function. As PGC-1 $\alpha$ is known to regulate mitochondrial function by modulating genes involved in mitochondrial biogenesis and dynamics [6], we focused on the PGC-1 $\alpha$ signaling pathway in the regulation of mitochondrial biogenesis and dynamics by DM in Dex-induced muscle atrophy models.

Mitochondrial biogenesis is important for determining mitochondrial content and function, and compounds capable of stimulating mitochondrial biogenesis have shown potential as treatments for a wide range of diseases [56]. Quercetin and resveratrol can promote mitochondrial biogenesis and attenuate mitochondrial dysfunction in skeletal muscle during disuse-induced muscle atrophy $[14,57]$. Our previous study suggested that DM improved mitochondrial biogenesis in skeletal muscle via upregulation of the PGC$1 \alpha$ signaling pathway during hypobaric hypoxia conditions [25]. In the present study, we found that DM enhanced mitochondrial biogenesis via activation of the PGC-1 $\alpha /$ TFAM signaling pathway, which was closely associated with the restoration of mtDNA content and mitochondrial mass in Dex-induced muscle atrophy models. Furthermore, mitochondria are dynamic organelles that constantly remodel their architecture through the fusion and fission processes. Mfn2, a mitochondrial fusion protein crucial for the maintenance of the mitochondrial network architecture, is induced during myogenesis and abundantly expressed in skeletal muscle [58]. Previous studies have suggested that $\mathrm{mfn} 2$ deficiency in muscles leads to mtDNA depletion, impaired OXPHOS, decreased mitochondrial membrane potential, and oxidative stress [59-61]. Moreover, muscle mfn2 protein levels were repressed in several catabolic conditions [62-65]. Lokireddy et al. showed that Dex increased mfn2 degradation in myotubes [66], and Liu et al. found that Dex decreased mfn2 levels in the TA muscles of mice [12], while Troncoso et al. reported that Dex had no influence on mfn2 expression in myotubes [67]. Our results suggested that Dex significantly decreased mfn2 expression levels in skeletal muscle both in vivo and in vitro. DM promoted mitochondrial fusion as shown by the restoration of $\mathrm{mfn} 2$ expression. Furthermore, PGC- $1 \alpha$ has been shown to induce the expression of $\mathrm{mfn} 2$ in muscle cells [68]; thus, DM might upregulate $\mathrm{mfn} 2$ expression through the activation of PGC- $1 \alpha$. As expected, in vitro PGC- $1 \alpha$ siRNA transfection markedly inhibited DM-induced mfn2 expression, suggesting that DM activated the PGC-1 $\alpha$ / $\mathrm{mfn} 2$ signaling pathway. We also evaluated the expression of the key mitochondrial fission protein drp1. The results showed that Dex increased drp1 levels in vitro, while it had no significant effect on drp1 levels in vivo. DM blocked Dex-mediated increased drp1 expression in vitro, but it had no effect on drp1 expression in vivo. Although there are differences regarding the effects of Dex or DM on drp1 expression in the whole muscle lysates between in vivo and in vitro studies, the levels of drp1 in the mitochondrial subfractions were increased by Dex but were decreased by DM both in vivo and in vitro, suggesting that DM attenuated Dex-mediated elevated mitochondrial fission via the inhibition of drp1 recruitment to the mitochondria. Additionally, we found that in vitro PGC- $1 \alpha$ siRNA transfection had no effect on drp1 expression both in the whole muscle lysates and the mitochondrial subfractions. The present study further demonstrated that PGC- $1 \alpha$ siRNA, TFAM siRNA and mfn2 siRNA transfection abolished the protective effects of DM on mitochondrial architecture, mtDNA content and mitochondrial function, suggesting enhanced mitochondrial biogenesis through the PGC-1 $\alpha /$ TFAM signaling pathway and improved mitochondrial dynamics through the PGC- $1 \alpha / \mathrm{mfn} 2$ signaling pathway as likely explanations for the restoration of mitochondrial function in Dex-induced muscle atrophy models treated with DM. In addition, there may be an interplay between mitochondrial biogenesis and mitochondrial dynamics [69]. In our study, we found that TFAM siRNA transfection resulted in discrete fragmented mitochondria, and $\mathrm{mfn} 2$ siRNA transfection reduced the mtDNA content and mitochondrial mass. Based on these findings, we speculated that DM exerted its action on mitochondrial function through the orchestration of mitochondrial biogenesis and mitochondrial dynamics via the activation of the PGC- $1 \alpha$ signaling pathway. 


\section{Cellular Physiology Cell Physiol Biochem 2018:49:758-779

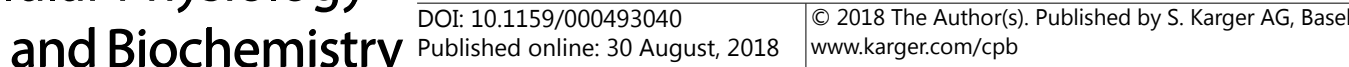

Huang et al.: Dihydromyricetin Improves Muscle Atrophy via PGC-1 $\alpha$

Oxidative stress is inseparably linked to mitochondrial dysfunction as mitochondria are both primary sources and targets of ROS[70]. The excessive mtROS production causes oxidative stress that increases oxidative damage to DNA, proteins and lipids not only in mitochondria but also in other cellular compartments, which can in turn exacerbate mitochondrial dysfunction and cause further damage to cells and tissues [71-73]. Several reports have indicated that Dex induces oxidative stress in osteoblast cells, hippocampal cells and thymocytes [74-76], but few studies have examined the effects of Dex on oxidative stress in skeletal muscle. We found that Dex markedly increased cellular ROS levels and caused oxidative damage to proteins and lipids in skeletal muscle, which were notably blocked by DM treatment. The observed protective effects of DM on Dex-induced oxidative stress might be attributed to the decreases in mtROS generation. In addition, given that DM has powerful antioxidant and free radical scavenging capabilities [17] and PGC-1 $\alpha$ is also a key factor that regulates the gene expression of antioxidant enzymes [77], it is possible that DM inhibits oxidative stress by directly scavenging excessive ROS or by increasing antioxidant enzyme activities, which could contribute to the improvement of mitochondrial function. Further experiments should be performed to clarify the mechanisms responsible for the protective effects of DM on mitochondrial dysfunction and oxidative stress in Dex-induced muscle atrophy models.

Previous studies suggested that mitochondrial dysfunction is involved in the activation of proteolytic systems [7], and the muscle proteins modified by oxidative stress are prone to proteolysis [78]. In addition, the activated proteolytic systems can in turn induce the breakdown of mitochondrial proteins and exacerbate mitochondrial dysfunction, which further accelerate the proteolysis of skeletal muscle proteins [12]. In this study, we focused on the ubiquitin-proteasome system, the main regulatory mechanism of protein degradation in skeletal muscle. The results indicated that DM blocked FoxO3a-mediated activation of ubiquitin-proteasome dependent proteolytic pathway by improving mitochondrial function. Moreover, previous reports demonstrated that muscle wasting stimuli resulted in enhanced FoxO3a-mediated activation of Mul1, which in turn ubiquitinates and degrades mfn2, leading to mitochondrial dysfunction and muscle atrophy [66]. Thus, DM might improve mitochondrial function through the inhibition of FoxO3a. In addition, PGC- $1 \alpha$ was shown to repress FoxO3a-dependent transcription of atrophy-specific genes [79]. Therefore, DM may attenuate Dex-induced muscle atrophy independent of its action on mitochondrial function.

Muscle mass is determined by both the rates of protein synthesis and degradation [80]. Thus, we also investigated the effects of DM on the Akt/mTOR signaling pathway, which plays a pivotal role in activating protein synthesis. The repression of the Akt/mTOR pathway by Dex was alleviated by DM, suggesting that DM was also effective in attenuating the reduced protein synthesis. Akt signaling has been shown to repress the expression of transcription factors in the FoxO family and then block the protein degradation pathways [80]. Moreover, mTOR signaling could be also directly inhibited by FoxO proteins [81]. Therefore, the protein synthesis and degradation processes are intimately connected, and DM improved protein metabolism through FoxO3a- and Akt/mTOR-dependent mechanisms. Moreover, PGC-1 $\alpha$ siRNA, TFAM siRNA and mfn2 siRNA transfection inhibited the DM-induced activation of the Akt/mTOR signaling pathway. Thus, the ability of DM to counteract the decreased protein synthesis was closely linked to its ability to improve mitochondrial function. Furthermore, emerging studies have indicated that mTOR stimulates mitochondrial biogenesis and regulates mitochondrial function [82]. Therefore, DM may prevent Dex-induced muscle atrophy by coordinating protein synthesis and mitochondrial function. Further experiments should be performed to explore additional mechanisms responsible for the protective effects of DM on Dex-induced muscle atrophy.

Although the present study suggested that DM effectively attenuated Dex-induced muscle atrophy in laboratory studies, given that muscle atrophy is a complex process that occurs as a consequence of multiple and interrelated stressors [83], it is necessary to investigate the effects of DM on other types of muscle atrophy as well as the underlying mechanisms. Moreover, in view of the good efficacy and safe properties of DM, DM-containing capsules are sold in the US as a nutraceutical supplement to prevent alcohol hangovers [84], and our previous randomized controlled clinical trial suggested that DM could improve liver steatosis in patients without any adverse events [85]. These studies demonstrated that DM may be developed as innovative and effective therapeutic agents. Future clinical randomized studies are needed to investigate whether DM has anti-muscle atrophy effects. 


\section{Cellular Physiology Cell Physiol Biochem 2018:49:758-779

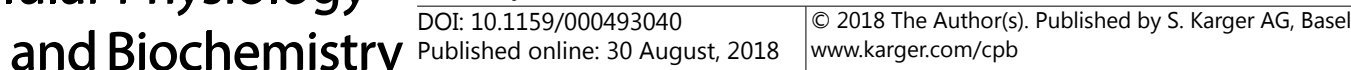

Huang et al.: Dihydromyricetin Improves Muscle Atrophy via PGC-1 $\alpha$

\section{Conclusion}

In summary, by stimulating mitochondrial biogenesis through the PGC- $1 \alpha /$ TFAM signaling pathway and promoting mitochondrial fusion through the PGC- $1 \alpha / \mathrm{mfn} 2$ signaling pathway, DM restored mitochondrial function, leading to improvements in protein metabolism and ultimately contributing to the inhibition of Dex-induced muscle atrophy. The present study proposes a new mechanism whereby DM attenuates Dex-induced muscle atrophy by improving mitochondrial function via upregulation of the PGC- $1 \alpha$ signaling pathway. These findings may help develop efficient therapeutic approaches for counteracting Dex-induced muscle atrophy.

\section{Acknowledgements}

This work was supported by the National Natural Science Foundation of China (No. 81573131; No.81773408), the key projects of the "Thirteenth Five-Year Plan" for Medical Science Development of PLA, China (BWS13J034), and the Research Fund of the Third Military Medical University (2016XYY03).

\section{Disclosure Statement}

The authors have no conflicts of interest to declare.

\section{References}

1 Frontera WR, Ochala J: Skeletal muscle: A brief review of structure and function. Calcif Tissue Int 2015;96:183-195.

2 Yang J: Enhanced skeletal muscle for effective glucose homeostasis. Prog Mol Biol Transl Sci 2014;121:133163.

3 Bonaldo P, Sandri M: Cellular and molecular mechanisms of muscle atrophy. Dis Model Mech 2013;6:25-39.

4 Schakman 0, Kalista S, Barbe C, Loumaye A, Thissen JP: Glucocorticoid-induced skeletal muscle atrophy. Int J Biochem Cell Biol 2013;45:2163-2172.

5 Umeki D, Ohnuki Y, Mototani Y, Shiozawa K, Suita K, Fujita T, Nakamura Y, Saeki Y, Okumura S: Protective effects of clenbuterol against Dexamethasone-Induced masseter muscle atrophy and myosin heavy chain transition. Plos One 2015;10:e128263.

6 Romanello V, Sandri M: Mitochondrial quality control and muscle mass maintenance. Front Physiol 2015;6:422.

7 Romanello V, Sandri M: Mitochondrial biogenesis and fragmentation as regulators of muscle protein degradation. Curr Hypertens Rep 2010;12:433-439.

-8 Marzetti E, Hwang JC, Lees HA, Wohlgemuth SE, Dupont-Versteegden EE, Carter CS, Bernabei R, Leeuwenburgh C: Mitochondrial death effectors: Relevance to sarcopenia and disuse muscle atrophy. Biochim Biophys Acta 2010;1800:235-244.

-9 Powers SK, Wiggs MP, Duarte JA, Zergeroglu AM, Demirel HA: Mitochondrial signaling contributes to disuse muscle atrophy. Am J Physiol Endocrinol Metab 2012;303:E31-E39.

10 Romanello V, Guadagnin E, Gomes L, Roder I, Sandri C, Petersen Y, Milan G, Masiero E, Del PP, Foretz M, Scorrano L, Rudolf R, Sandri M: Mitochondrial fission and remodelling contributes to muscle atrophy. Embo J 2010;29:1774-1785.

11 Boengler K, Kosiol M, Mayr M, Schulz R, Rohrbach S: Mitochondria and ageing: Role in heart, skeletal muscle and adipose tissue. J Cachexia Sarcopenia Muscle 2017;8:349-369.

12 Liu J, Peng Y, Wang X, Fan Y, Qin C, Shi L, Tang Y, Cao K, Li H, Long J, Liu J: Mitochondrial Dysfunction Launches Dexamethasone-Induced Skeletal Muscle Atrophy via AMPK/FOXO3 Signaling. Mol Pharm 2016;13:73-84.

13 Wang X, Li H, Zheng A, Yang L, Liu J, Chen C, Tang Y, Zou X, Li Y, Long J, Liu J, Zhang Y, Feng Z: Mitochondrial dysfunction-associated OPA1 cleavage contributes to muscle degeneration: Preventative effect of hydroxytyrosol acetate. Cell Death Dis 2014;5:e1521. 


\section{Cellular Physiology Cell Physiol Biochem 2018;49:758-779 \begin{tabular}{l|l|l} 
and Biochemistry & $\begin{array}{l}\text { DOI: 10.1159/000493040 } \\
\text { Published onlIne: 30 August, } 2018\end{array}$ & $\begin{array}{l}\text { C) 2018 The Author(s). Published by S. Karger AG, Basel } \\
\text { www.karger.com/cpb }\end{array}$ \\
\hline
\end{tabular}}

14 Mukai R, Matsui N, Fujikura Y, Matsumoto N, Hou DX, Kanzaki N, Shibata H, Horikawa M, Iwasa K, Hirasaka K, Nikawa T, Terao J: Preventive effect of dietary quercetin on disuse muscle atrophy by targeting mitochondria in denervated mice. J Nutr Biochem 2016;31:67-76.

15 Hou X, Tong Q Wang W, Xiong W, Shi C, Fang J: Dihydromyricetin protects endothelial cells from hydrogen peroxide-induced oxidative stress damage by regulating mitochondrial pathways. Life Sci 2015;130:38-46.

16 Hou X, Tong Q Wang W, Xiong W, Shi C, Fang J: Dihydromyricetin protects endothelial cells from hydrogen peroxide-induced oxidative stress damage by regulating mitochondrial pathways. Life Sci 2015;130:38-46.

17 Ye L, Wang H, Duncan SE, Eigel WN, O’Keefe SF: Antioxidant activities of Vine Tea (Ampelopsis grossedentata) extract and its major component dihydromyricetin in soybean oil and cooked ground beef. Food Chem 2015;172:416-422.

18 Hou XL, Tong Q Wang WQ, Shi CY, Xiong W, Chen J, Liu X, Fang JG: Suppression of Inflammatory Responses by Dihydromyricetin, a Flavonoid from Ampelopsis grossedentata, via Inhibiting the Activation of NFkappaB and MAPK Signaling Pathways. J Nat Prod 2015;78:1689-1696.

19 Zhao Z, Yin JQ, Wu MS, Song G, Xie XB, Zou C, Tang Q, Wu Y, Lu J, Wang Y, Wang J, Kang T, Jia Q, Shen J: Dihydromyricetin activates AMP-activated protein kinase and P38(MAPK) exerting antitumor potential in osteosarcoma. Cancer Prev Res (Phila) 2014;7:927-938.

-20 Liu P, Zou D, Chen K, Zhou Q, Gao Y, Huang Y, Zhu J, Zhang Q Mi M: Dihydromyricetin improves hypobaric Hypoxia-Induced memory impairment via modulation of SIRT3 signaling. Mol Neurobiol 2016;53:72007212.

21 Qiu P, Dong Y, Li B, Kang XJ, Gu C, Zhu T, Luo YY, Pang MX, Du WF, Ge WH: Dihydromyricetin modulates p62 and autophagy crosstalk with the Keap-1/Nrf2 pathway to alleviate ethanol-induced hepatic injury. Toxicol Lett 2017;274:31-41.

22 Shi L, Zhang T, Zhou Y, Zeng X, Ran L, Zhang Q, Zhu J, Mi M: Dihydromyricetin improves skeletal muscle insulin sensitivity by inducing autophagy via the AMPK-PGC-1alpha-Sirt3 signaling pathway. Endocrine 2015;50:378-389.

23 Zhou Q, Gu Y, Lang H, Wang X, Chen K, Gong X, Zhou M, Ran L, Zhu J, Mi M: Dihydromyricetin prevents obesity-induced slow-twitch-fiber reduction partially via FLCN/FNIP1/AMPK pathway. Biochim Biophys Acta 2017;1863:1282-1291.

24 Zhou Q Chen K, Liu P, Gao Y, Zou D, Deng H, Huang Y, Zhang Q Zhu J, Mi M: Dihydromyricetin stimulates irisin secretion partially via the PGC-1alpha pathway. Mol Cell Endocrinol 2015;412:349-357.

25 Zou D, Chen K, Liu P, Chang H, Zhu J, Mi M: Dihydromyricetin improves physical performance under simulated high altitude. Med Sci Sports Exerc 2014;46:2077-2084.

26 Liu J, Peng Y, Cui Z, Wu Z, Qian A, Shang P, Qu L, Li Y, Liu J, Long J: Depressed mitochondrial biogenesis and dynamic remodeling in mouse tibialis anterior and gastrocnemius induced by 4 -week hindlimb unloading. Iubmb Life 2012;64:901-910.

27 Theilen NT, Kunkel GH, Tyagi SC: The role of exercise and TFAM in preventing skeletal muscle atrophy. J Cell Physiol 2017;232:2348-2358.

28 Russell AP, Wada S, Vergani L, Hock MB, Lamon S, Leger B, Ushida T, Cartoni R, Wadley GD, Hespel P, Kralli A, Soraru G, Angelini C, Akimoto T: Disruption of skeletal muscle mitochondrial network genes and miRNAs in amyotrophic lateral sclerosis. Neurobiol Dis 2013;49:107-117.

29 Cannavino J, Brocca L, Sandri M, Grassi B, Bottinelli R, Pellegrino MA: The role of alterations in mitochondrial dynamics and PGC-1alpha over-expression in fast muscle atrophy following hindlimb unloading. J Physiol 2015;593:1981-1995.

30 Wenz T, Rossi SG, Rotundo RL, Spiegelman BM, Moraes CT: Increased muscle PGC-1alpha expression protects from sarcopenia and metabolic disease during aging. Proc Natl Acad Sci U S A 2009;106:2040520410.

-31 Sandri M, Lin J, Handschin C, Yang W, Arany ZP, Lecker SH, Goldberg AL, Spiegelman BM: PGC-1alpha protects skeletal muscle from atrophy by suppressing FoxO3 action and atrophy-specific gene transcription. Proc Natl Acad Sci U S A 2006;103:16260-16265.

32 Geng T, Li P, Yin X, Yan Z: PGC-1alpha promotes nitric oxide antioxidant defenses and inhibits FOXO signaling against cardiac cachexia in mice. Am J Pathol 2011;178:1738-1748.

-33 Qin W, Pan J, Wu Y, Bauman WA, Cardozo C: Protection against dexamethasone-induced muscle atrophy is related to modulation by testosterone of FOXO1 and PGC-1alpha. Biochem Biophys Res Commun 2010;403:473-478.

34 Rahnert JA, Zheng B, Hudson MB, Woodworth-Hobbs ME, Price SR: Glucocorticoids alter CRTCCREB signaling in muscle cells: Impact on PGC-1alpha expression and atrophy markers. Plos One 2016;11:e159181.

-35 Liu J, Peng Y, Feng Z, Shi W, Qu L, Li Y, Liu J, Long J: Reloading functionally ameliorates disuse-induced muscle atrophy by reversing mitochondrial dysfunction, and similar benefits are gained by administering a combination of mitochondrial nutrients. Free Radic Biol Med 2014;69:116-128. 


\section{Cellular Physiology Cell Physiol Biochem 2018;49:758-779 \begin{tabular}{l|l|l}
\hline DOI: 10.1159/000493040 & $\begin{array}{l}\text { C } 2018 \text { The Author(s). Published by S. Karger AG, Basel } \\
\text { www.karger.com/cpb }\end{array}$
\end{tabular}

-36 Zhou X, Chen M, Zeng X, Yang J, Deng H, Yi L, Mi MT: Resveratrol regulates mitochondrial reactive oxygen species homeostasis through Sirt3 signaling pathway in human vascular endothelial cells. Cell Death Dis 2014;5:e1576.

37 Menconi M, Gonnella P, Petkova V, Lecker S, Hasselgren PO: Dexamethasone and corticosterone induce similar, but not identical, muscle wasting responses in cultured L6 and C2C12 myotubes. J Cell Biochem 2008;105:353-364.

-38 Carrasco-Pozo C, Tan KN, Reyes-Farias M, De La Jara N, Ngo ST, Garcia-Diaz DF, Llanos P, Cires MJ, Borges $\mathrm{K}$ : The deleterious effect of cholesterol and protection by quercetin on mitochondrial bioenergetics of pancreatic beta-cells, glycemic control and inflammation: In vitro and in vivo studies. Redox Biol 2016;9:229-243.

39 Wang W, Fang H, Groom L, Cheng A, Zhang W, Liu J, Wang X, Li K, Han P, Zheng M, Yin J, Wang W, Mattson MP, Kao JP, Lakatta EG, Sheu SS, Ouyang K, Chen J, Dirksen RT, Cheng H: Superoxide flashes in single mitochondria. Cell 2008;134:279-290.

40 Yang KC, Bonini MG, Dudley SJ: Mitochondria and arrhythmias. Free Radic Biol Med 2014;71:351-361.

-41 Li XX, He GR, Mu X, Xu B, Tian S, Yu X, Meng FR, Xuan ZH, Du GH: Protective effects of baicalein against rotenone-induced neurotoxicity in PC12 cells and isolated rat brain mitochondria. Eur J Pharmacol 2012;674:227-233.

42 Sanchez AM, Candau RB, Bernardi H: FoxO transcription factors: Their roles in the maintenance of skeletal muscle homeostasis. Cell Mol Life Sci 2014;71:1657-1671.

43 Bodine SC, Stitt TN, Gonzalez M, Kline WO, Stover GL, Bauerlein R, Zlotchenko E, Scrimgeour A, Lawrence JC, Glass DJ, Yancopoulos GD: Akt/mTOR pathway is a crucial regulator of skeletal muscle hypertrophy and can prevent muscle atrophy in vivo. Nat Cell Biol 2001;3:1014-1019.

44 Tavares MR, Pavan IC, Amaral CL, Meneguello L, Luchessi AD, Simabuco FM: The S6K protein family in health and disease. Life Sci 2015;131:1-10.

45 Iizuka K, Machida T, Hirafuji M: Skeletal muscle is an endocrine organ. J Pharmacol Sci 2014;125:125-131.

-46 Cohen S, Nathan JA, Goldberg AL: Muscle wasting in disease: Molecular mechanisms and promising therapies. Nat Rev Drug Discov 2015;14:58-74.

47 Waltenberger B, Mocan A, Smejkal K, Heiss EH, Atanasov AG: Natural products to counteract the epidemic of cardiovascular and metabolic disorders. Molecules 2016;21.

48 Dwyer JT, Peterson J: Tea and flavonoids: Where we are, where to go next. Am J Clin Nutr 2013;98:1611S-1618S.

49 Le NH, Kim CS, Park T, Park JH, Sung MK, Lee DG, Hong SM, Choe SY, Goto T, Kawada T, Yu R: Quercetin protects against obesity-induced skeletal muscle inflammation and atrophy. Mediators Inflamm 2014;2014:834294.

50 Mukai R, Nakao R, Yamamoto H, Nikawa T, Takeda E, Terao J: Quercetin prevents unloading-derived disused muscle atrophy by attenuating the induction of ubiquitin ligases in tail-suspension mice. J Nat Prod 2010;73:1708-1710.

-51 Emanuele E, Bertona M, Pareja-Galeano H, Fiuza-Luces C, Morales JS, Sanchis-Gomar F, Lucia A: Baicalin supplementation reduces serum biomarkers of skeletal muscle wasting and may protect against lean body mass reduction in cancer patients: Results from a pilot open-label study. Neuro Endocrinol Lett 2016;37:213-216.

52 Li B, Wan L, Li Y, Yu Q Chen P, Gan R, Yang Q Han Y, Guo C: Baicalin, a component of Scutellaria baicalensis, alleviates anorexia and inhibits skeletal muscle atrophy in experimental cancer cachexia. Tumour Biol 2014;35:12415-12425.

53 Wang H, Lai YJ, Chan YL, Li TL, Wu CJ: Epigallocatechin-3-gallate effectively attenuates skeletal muscle atrophy caused by cancer cachexia. Cancer Lett 2011;305:40-49.

54 Murata M, Nonaka H, Komatsu S, Goto M, Morozumi M, Yamada S, Lin IC, Yamashita S, Tachibana H: Delphinidin prevents muscle atrophy and upregulates miR-23a expression. J Agric Food Chem 2017;65:4550 .

55 Schakman 0, Gilson H, Thissen JP: Mechanisms of glucocorticoid-induced myopathy. J Endocrinol 2008;197:1-10.

56 Whitaker RM, Corum D, Beeson CC, Schnellmann RG: Mitochondrial biogenesis as a pharmacological target: A new approach to acute and chronic diseases. Annu Rev Pharmacol Toxicol 2016;56:229-249.

57 Momken I, Stevens L, Bergouignan A, Desplanches D, Rudwill F, Chery I, Zahariev A, Zahn S, Stein TP, Sebedio JL, Pujos-Guillot E, Falempin M, Simon C, Coxam V, Andrianjafiniony T, Gauquelin-Koch G, Picquet F, Blanc S: Resveratrol prevents the wasting disorders of mechanical unloading by acting as a physical exercise mimetic in the rat. Faseb J 2011;25:3646-3660. 


\section{Cellular Physiology Cell Physiol Biochem 2018;49:758-779 \begin{tabular}{l|l|l} 
and Biochemistry & $\begin{array}{l}\text { DOI: 10.1159/000493040 } \\
\text { Published onlIne: 30 August, } 2018\end{array}$ & $\begin{array}{l}\text { C) 2018 The Author(s). Published by S. Karger AG, Basel } \\
\text { www.karger.com/cpb }\end{array}$ \\
\hline
\end{tabular}}

Huang et al.: Dihydromyricetin Improves Muscle Atrophy via PGC-1 $\alpha$

58 Bach D, Pich S, Soriano FX, Vega N, Baumgartner B, Oriola J, Daugaard JR, Lloberas J, Camps M, Zierath JR, Rabasa-Lhoret R, Wallberg-Henriksson H, Laville M, Palacin M, Vidal H, Rivera F, Brand M, Zorzano A: Mitofusin-2 determines mitochondrial network architecture and mitochondrial metabolism. A novel regulatory mechanism altered in obesity. J Biol Chem 2003;278:17190-17197.

59 Vielhaber S, Debska-Vielhaber G, Peeva V, Schoeler S, Kudin AP, Minin I, Schreiber S, Dengler R, Kollewe K, Zuschratter W, Kornblum C, Zsurka G, Kunz WS: Mitofusin 2 mutations affect mitochondrial function by mitochondrial DNA depletion. Acta Neuropathol 2013;125:245-256.

60 Pich S, Bach D, Briones P, Liesa M, Camps M, Testar X, Palacin M, Zorzano A: The Charcot-Marie-Tooth type 2A gene product, Mfn2, up-regulates fuel oxidation through expression of OXPHOS system. Hum Mol Genet 2005;14:1405-1415.

61 Nie Q, Wang C, Song G, Ma H, Kong D, Zhang X, Gan K, Tang Y: Mitofusin 2 deficiency leads to oxidative stress that contributes to insulin resistance in rat skeletal muscle cells. Mol Biol Rep 2014;41:6975-6983.

62 Bach D, Naon D, Pich S, Soriano FX, Vega N, Rieusset J, Laville M, Guillet C, Boirie Y, Wallberg-Henriksson H, Manco M, Calvani M, Castagneto M, Palacin M, Mingrone G, Zierath JR, Vidal H, Zorzano A: Expression of Mfn2, the Charcot-Marie-Tooth neuropathy type 2A gene, in human skeletal muscle: Effects of type 2 diabetes, obesity, weight loss, and the regulatory role of tumor necrosis factor alpha and interleukin- 6 Diabetes 2005;54:2685-2693.

-63 White JP, Puppa MJ, Sato S, Gao S, Price RL, Baynes JW, Kostek MC, Matesic LE, Carson JA: IL-6 regulation on skeletal muscle mitochondrial remodeling during cancer cachexia in the ApcMin/+ mouse. Skelet Muscle 2012;2:14.

64 Hernandez-Alvarez MI, Thabit H, Burns N, Shah S, Brema I, Hatunic M, Finucane F, Liesa M, Chiellini C, Naon D, Zorzano A, Nolan JJ: Subjects with early-onset type 2 diabetes show defective activation of the skeletal muscle PGC-1\{alpha\}/Mitofusin-2 regulatory pathway in response to physical activity. Diabetes Care 2010;33:645-651.

65 Xi QL, Zhang B, Jiang Y, Zhang HS, Meng QY, Chen Y, Han YS, Zhuang QL, Han J, Wang HY, Fang J, Wu GH: Mitofusin-2 prevents skeletal muscle wasting in cancer cachexia. Oncol Lett 2016;12:4013-4020.

66 Lokireddy S, Wijesoma IW, Teng S, Bonala S, Gluckman PD, McFarlane C, Sharma M, Kambadur R: The ubiquitin ligase Mul1 induces mitophagy in skeletal muscle in response to muscle-wasting stimuli. Cell Metab 2012;16:613-624.

-67 Troncoso R, Paredes F, Parra V, Gatica D, Vasquez-Trincado C, Quiroga C, Bravo-Sagua R, Lopez-Crisosto C, Rodriguez AE, Oyarzun AP, Kroemer G, Lavandero S: Dexamethasone-induced autophagy mediates muscle atrophy through mitochondrial clearance. Cell Cycle 2014;13:2281-2295.

68 Soriano FX, Liesa M, Bach D, Chan DC, Palacin M, Zorzano A: Evidence for a mitochondrial regulatory pathway defined by peroxisome proliferator-activated receptor-gamma coactivator-1 alpha, estrogenrelated receptor-alpha, and mitofusin 2. Diabetes 2006;55:1783-1791.

69 Dorn GN, Vega RB, Kelly DP: Mitochondrial biogenesis and dynamics in the developing and diseased heart. Genes Dev 2015;29:1981-1991.

-70 Johri A, Chandra A, Beal MF: PGC-1alpha, mitochondrial dysfunction, and Huntington's disease. Free Radic Biol Med 2013;62:37-46.

71 Li XX, He GR, Mu X, Xu B, Tian S, Yu X, Meng FR, Xuan ZH, Du GH: Protective effects of baicalein against rotenone-induced neurotoxicity in PC12 cells and isolated rat brain mitochondria. Eur J Pharmacol 2012;674:227-233.

72 Kuwahara H, Horie T, Ishikawa S, Tsuda C, Kawakami S, Noda Y, Kaneko T, Tahara S, Tachibana T, Okabe M, Melki J, Takano R, Toda T, Morikawa D, Nojiri H, Kurosawa H, Shirasawa T, Shimizu T: Oxidative stress in skeletal muscle causes severe disturbance of exercise activity without muscle atrophy. Free Radic Biol Med 2010;48:1252-1262.

-73 Joseph AM, Adhihetty PJ, Wawrzyniak NR, Wohlgemuth SE, Picca A, Kujoth GC, Prolla TA, Leeuwenburgh C: Dysregulation of mitochondrial quality control processes contribute to sarcopenia in a mouse model of premature aging. Plos One 2013;8:e69327.

74 Inkielewicz-Stepniak I, Radomski MW, Wozniak M: Fisetin prevents fluoride- and dexamethasone-induced oxidative damage in osteoblast and hippocampal cells. Food Chem Toxicol 2012;50:583-589.

-75 You JM, Yun SJ, Nam KN, Kang C, Won R, Lee EH: Mechanism of glucocorticoid-induced oxidative stress in rat hippocampal slice cultures. Can J Physiol Pharmacol 2009;87:440-447.

-76 Yi J, Zhu R, Wu J, Wu J, Xia W, Zhu L, Jiang W, Xiang S, Tan Z: In vivo protective effect of betulinic acid on dexamethasone induced thymocyte apoptosis by reducing oxidative stress. Pharmacol Rep 2016;68:95100.

77 Kang C, Li JL: Role of PGC-1alpha signaling in skeletal muscle health and disease. Ann N Y Acad Sci 2012;1271:110-117.

78 Powers SK, Smuder AJ, Criswell DS: Mechanistic links between oxidative stress and disuse muscle atrophy. Antioxid Redox Signal 2011;15:2519-2528. 


\section{Cellular Physiology Cell Physiol Biochem 2018:49:758-779 \begin{tabular}{ll|l} 
DOI: 10.1159/000493040 & $\begin{array}{l}\text { O 2018 The Author(s). Published by S. Karger AG, Basel } \\
\text { www.karger.com/cpb }\end{array}$ \\
\hline
\end{tabular}}

Huang et al.: Dihydromyricetin Improves Muscle Atrophy via PGC-1 $\alpha$

79 Sandri M, Lin J, Handschin C, Yang W, Arany ZP, Lecker SH, Goldberg AL, Spiegelman BM: PGC-1alpha protects skeletal muscle from atrophy by suppressing FoxO3 action and atrophy-specific gene transcription. Proc Natl Acad Sci U S A 2006;103:16260-16265.

80 Schiaffino S, Dyar KA, Ciciliot S, Blaauw B, Sandri M: Mechanisms regulating skeletal muscle growth and atrophy. Febs J 2013;280:4294-4314.

-81 Chen CC, Jeon SM, Bhaskar PT, Nogueira V, Sundararajan D, Tonic I, Park Y, Hay N: FoxOs inhibit mTORC1 and activate Akt by inducing the expression of Sestrin3 and Rictor. Dev Cell 2010;18:592-604.

-82 Morita M, Gravel SP, Hulea L, Larsson O, Pollak M, St-Pierre J, Topisirovic I: MTOR coordinates protein synthesis, mitochondrial activity and proliferation. Cell Cycle 2015;14:473-480.

83 Bodine SC, Baehr LM: Skeletal muscle atrophy and the E3 ubiquitin ligases MuRF1 and MAFbx/atrogin-1 Am J Physiol Endocrinol Metab 2014;307:E469-E484.

84 Wang C, Xiong W, Reddy PS, Fang J, Calvin SC: Solid-state characterization of optically pure (+) Dihydromyricetin extracted from Ampelopsis grossedentata leaves. Int J Pharm 2016;511:245-252.

85 Chen S, Zhao X, Wan J, Ran L, Qin Y, Wang X, Gao Y, Shu F, Zhang Y, Liu P, Zhang Q Zhu J, Mi M: Dihydromyricetin improves glucose and lipid metabolism and exerts anti-inflammatory effects in nonalcoholic fatty liver disease: A randomized controlled trial. Pharmacol Res 2015;99:74-81. 